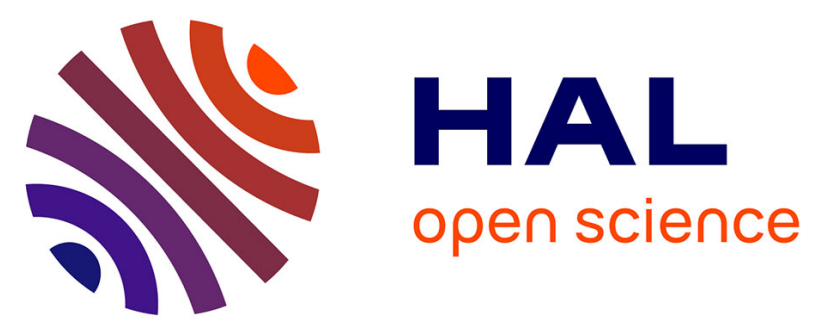

\title{
The ability of MM5 to simulate ice clouds : systematic comparison between simulated and measured fluxes and lidar/radar profiles at the SIRTA atmospheric observatory.
}

\author{
Marjolaine Chiriaco, Robert Vautard, Hélène Chepfer, Martial Haeffelin, J.
}

Dudhia, Y. Wanhendrick, Yohann Morille, Alain Protat

\section{To cite this version:}

Marjolaine Chiriaco, Robert Vautard, Hélène Chepfer, Martial Haeffelin, J. Dudhia, et al.. The ability of MM5 to simulate ice clouds: systematic comparison between simulated and measured fluxes and lidar/radar profiles at the SIRTA atmospheric observatory.. Monthly Weather Review, 2006, 134 (3), pp.897-918. 10.1175/MWR3102.1 . hal-00159781

\author{
HAL Id: hal-00159781 \\ https://hal.science/hal-00159781
}

Submitted on 22 Dec 2020

HAL is a multi-disciplinary open access archive for the deposit and dissemination of scientific research documents, whether they are published or not. The documents may come from teaching and research institutions in France or abroad, or from public or private research centers.
L'archive ouverte pluridisciplinaire HAL, est destinée au dépôt et à la diffusion de documents scientifiques de niveau recherche, publiés ou non, émanant des établissements d'enseignement et de recherche français ou étrangers, des laboratoires publics ou privés. 


\title{
The Ability of MM5 to Simulate Ice Clouds: Systematic Comparison between Simulated and Measured Fluxes and Lidar/Radar Profiles at the SIRTA Atmospheric Observatory
}

\author{
M. Chiriaco, R. Vautard, H. Chepfer, and M. Haeffelin \\ Laboratoire de Météorologie Dynamique, Institut Pierre Simon Laplace, Palaiseau, France \\ J. DUDHIA \\ NCAR, Boulder, Colorado \\ Y. WANHERDRICK AND Y. MORILlE \\ Laboratoire de Météorologie Dynamique, Institut Pierre Simon Laplace, Palaiseau, France \\ A. Protat \\ Centre d'études des Environnements Terrestre et Planétaires, Institut Pierre Simon Laplace, Velizy, France \\ (Manuscript received 31 January 2005, in final form 5 August 2005)

\begin{abstract}
The ability of the fifth-generation Pennsylvania State University-NCAR Mesoscale Model (MM5) to simulate midlatitude ice clouds is evaluated. Model outputs are compared to long-term meteorological measurements by active (radar and lidar) and passive (infrared and visible fluxes) remote sensing collected at an atmospheric observatory near Paris, France. The goal is to understand which of four microphysical schemes is best suited to simulate midlatitude ice clouds. The methodology consists of simulating instrument observables from the model outputs without any profile inversion, which allows the authors to use fewer assumptions on microphysical and optical properties of ice particles.

Among the four schemes compared in the current study, the best observation-to-simulations scores are obtained with Reisner et al. provided that the particles' sedimentation velocity from Heymsfield and Donner is used instead of that originally proposed. For this last scheme, the model gives results close to the measurements for clouds with medium optical depth of typically 1 to 3 , whatever the season. In this configuration, MM5 simulates the presence of midlatitude ice clouds in more than $65 \%$ of the authors' selection of observed cloud cases. In $35 \%$ of the cases, the simulated clouds are too persistent whatever the microphysical scheme and tend to produce too much solid water (ice and snow) and not enough liquid water.
\end{abstract}

\section{Introduction}

Ice clouds play a major role in the radiative energy budget of the earth-atmosphere system (Liou 1986). Their radiative effect is governed primarily by the equilibrium between their albedo effect and their greenhouse effect. Both macrophysical and microphysical

Corresponding author address: Marjolaine Chiriaco, Laboratoire de Météorologie Dynamique/IPSL, Ecole Polytechnique, 91128 Palaiseau CEDEX, France.

E-mail: chiriaco@lmd.polytechnique.fr properties of ice clouds regulate this equilibrium. To quantify the effect of these clouds onto climate and weather systems, their global coverage, altitude, temperature, vertical structure, spatial heterogeneities, and life duration must be properly characterized and accounted for in atmospheric models. Additionally, the ice water content and its spatial distribution are critical to the global radiative effect of ice clouds. One of the main uncertainties on the radiative impact of ice clouds is due to the poor knowledge of the natural variability of their microphysical properties, such as ice crystal size and shape. 
The difficulty in representing ice clouds in general circulation models is not only due to the microphysics itself but also to the model resolution, generally much coarser than the cloud size. This problem is less acute in mesoscale models where the widest clouds can be represented explicitly but another uncertainty could come from the difficulty in representing the thermodynamical structure that creates the conditions for the existence of clouds and associated microphysical properties. The main goal of the present study is to assess the ability of several microphysics schemes to simulate ice clouds in a mesoscale model, the fifth-generation Pennsylvania State University-National Center for Atmospheric Research (PSU-NCAR) Mesoscale Model (MM5; Dudhia 1993).

There are two principal classes of microphysical schemes: the first one, called "bin," is the most detailed and allows the evolution of cloud particle dimensions to be calculated, using the actual theoretical and experimental knowledge. It is used for studies at the cloud scale (Lin 1997; Khvorostyanov and Sassen 1998) in order to understand the formation, structure, and dissipation of clouds (Xu et al. 1992; Krueger et al. 1995a,b). The second class of microphysical scheme (called "bulk") is developed for the study of the ice water content (IWC) and evolution of number of cloud particles. This type of scheme is coupled with dynamical and radiative schemes in the mesoscale model in order to study the cloud development (Levkov et al. 1992, 1998; Heckman and Cotton 1993; Walko et al. 1995; Lafore et al. 1998; Guichard et al. 2003). The current study evaluates schemes of this second category.

Verifying model ice cloud properties versus real ice cloud ones is not an easy task, and has been attempted in several field campaigns using in situ airborne measurements or remote sensing measurements such as the Improvement of Microphysical Parameterization through Observational Verification Experiment (IMPROVE). However, the natural variability of clouds and their associated properties makes it illusory to fully evaluate a microphysical scheme using in situ observations in a few intensive case studies. Nevertheless, a statistical long-term observations approach could also show biases that are more difficult to explain.

In this paper, we use remote sensing measurements obtained from the Site Instrumental de Recherche par Télédétection Atmosphérique (SIRTA; http://sirta.lmd. polytechnique.fr) ground-based atmospheric observatory. Lidar and radar observations taken over 18 months are used for statistical comparison with the model. During this period of time, 62 days containing parts of ice clouds have been analyzed.
The evaluation of cloud properties in mesoscale and global-scale models using remote sensing observations has been carried out in the literature using two complementary approaches (Doutriaux-Boucher and Quaas 2004; Mathieu et al. 2004, manuscript submitted to Geophys. Res. Lett.; Bony et al. 2004; Jakob et al. 2004): the first approach (observations to model) is to compare cloud parameters derived from satellite or groundbased remote sensing observations with those directly held in the model; the second one (model to observations) is to simulate the raw observations using the model outputs. The second method generally involves fewer assumptions than the first one. In this paper, we use the "model-to-observations" approach by simulating the lidar and radar signals from MM5 outputs. Other more classical variables such as shortwave and longwave radiative fluxes are also used.

Four microphysical schemes are evaluated in this study, including that proposed by Reisner et al. (1998) with original or modified parameterizations of particle terminal fall velocities (Zurovac-Jevtic and Zhang 2003; Heymsfield and Donner 1990) and the simplified Dudhia (1989) scheme. Section 2 describes the MM5 simulations, and section 3 describes the observations. The method of comparison is developed in section 4. A case study (section 5) illustrates the approach before the full statistical analysis (section 6). Discussion and conclusions follow in section 7 .

\section{MM5 model simulations}

\section{a. MM5 model configuration and simulations}

The MM5 model domain covers northern France and consists of two nested grids, the coarsest resolution being $15 \mathrm{~km}$ and the finest $5 \mathrm{~km}$. The two-way nesting method is used. The finest grid, which is that from which cloud fields are eventually extracted for the present study, covers about $200 \mathrm{~km} \times 200 \mathrm{~km}$ around the city of Paris. Figure 1 shows the largest domain centered on Paris and the SIRTA ground-based site, located $25 \mathrm{~km}$ south of Paris.

At the boundaries of the coarse domain, MM5 is forced by the National Centers for Environmental Prediction (NCEP) $1^{\circ} \times 1^{\circ}$ analyses provided every $6 \mathrm{~h}$. In order for the model to stay close to the analyses, the nudging procedure is applied to wind and temperature with a relaxation time of $20000 \mathrm{~s}$, for both domains. This nudging procedure forces the model thermodynamical conditions to be equivalent to the real (i.e., observed) ones. It is then possible to focus the comparison between observations and simulations on aspects directly related to the microphysical schemes, as 


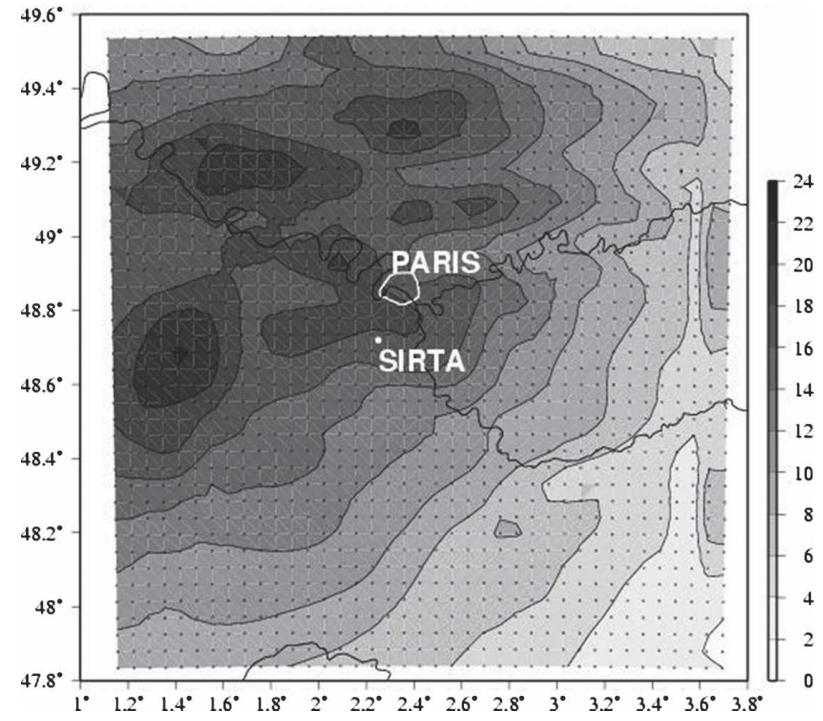

FIG. 1. Total water column $\left(\mathrm{g} \mathrm{m}^{-2}\right)$ for 0900 UTC 10 Oct 2003 around SIRTA. The domain is about $500 \times 500 \mathrm{~km}^{2}$.

they are mostly responsible for the differences between simulations and observations. Nevertheless, parameterizations of subgrid-scale turbulence and convection processes cannot be forced by this nudging procedure as they play a fundamental role in cloud simulations. Hence, they can also have an impact on the difference between simulations and observations. The humidity field does not undergo nudging in order to not perturb the water variables.

We choose a vertical setup of 61 layers from the ground to the $100-\mathrm{hPa}$ top surface. Model layers are about 200-400 $\mathrm{m}$ thick in the upper troposphere where ice clouds reside. The maximum height of the model is about $14 \mathrm{~km}$, typically above the altitude of the tropopause at midlatitude, so just above the limit of clouds. Outputs are saved with an hourly time step. For each cloud case, a 30-h simulation is carried out, starting on the previous day at 1800 UTC and ending at midnight.

\section{b. Parameterizations}

We use the classical parameterizations of the model that are proposed as options in the MM5 configuration file, with the following modifications. Regarding convection, the Grell (1993) scheme is used without modification. Turbulence in the planetary boundary layer is parameterized using the Medium Range Forecast scheme (MRFPBL), mostly borrowed from the Troën and Mahrt (1986) approach. According to Liu et al. (2004), the original calculation of the friction velocity uses an unrealistic convective velocity parameterization. As suggested by Liu et al., the convective velocity formulation is replaced by the Beljaars (1994) formulation. The Noah land surface model is used (Chen and Dudhia 2001).

Atmospheric radiation transfer uses the optional NCAR Community Climate Model (CCM2; Hack et al. 1993) scheme, with a modification on assumed fixed effective ice particle diameter of $35 \mu \mathrm{m}$ instead of 14.6 $\mu \mathrm{m}$ in the original routine, which is a more realistic value (Liou 2002). Note that in MM5 version 3.6.2 this radiation scheme does not use prognostic ice particle diameter, which could be deduced from ice content and particle number.

In this study, our intention is to evaluate and compare several microphysical schemes. Four schemes that are available as MM5 options or variants are used (Table 1). Three of them (A, B, and C) are derived from the Reisner et al. (1998) scheme, but differ from each other by the terminal fall $V_{t}\left(\mathrm{~m} \mathrm{~s}^{-1}\right)$ formulation. The last one (D) is the so-called simple ice scheme (Dudhia 1989). For the first three schemes, mass contents for liquid water cloud, ice cloud, rain, snow, graupel, and ice number concentration are prognostic variables. That helps diagnose a time-varying mean particle size. For rain, snow, and graupel, size distributions are assumed, and ice multiplication processes are parameterized. The last scheme has no mixed-phase processes or supercooled water, and snow is assumed to melt immediately at the melting layer. For this scheme, the

TABLE 1. Description of the four microphysical schemes used in MM5.

\begin{tabular}{|c|c|c|}
\hline $\begin{array}{l}\text { Name of the } \\
\text { microphysics scheme }\end{array}$ & Reference of the parameterization & Ice particle sedimentation velocity \\
\hline A & Reisner et al. (1998) option 5- $\mathrm{N}_{\text {ice }}$ predicted & $\begin{array}{l}\text { Zurovac-Jevtic and Zhang }(2003): V_{\text {fall }}=a\left(q_{\text {ice }} \rho_{\text {air }}\right)^{b} \\
\text { if } q_{\text {ice }} \rho_{\text {air }} \leq 4.8 \times 10^{-6}: a=180.65 \text { and } b=0.52 \\
\text { otherwise } a=3.13 \text { and } b=0.19\end{array}$ \\
\hline $\mathrm{B}$ & Reisner et al. (1998) option 5- $\mathrm{N}_{\text {ice }}$ predicted & $\begin{array}{l}\text { Heymsfield and Donner (1990): } V_{\text {fall }}=a\left(q_{\text {ice }} \rho_{\text {air }}\right)^{b} \\
\quad a=3.29 \text { and } b=0.16\end{array}$ \\
\hline $\mathrm{C}$ & Reisner et al. (1998) option 5- $\mathrm{N}_{\text {ice }}$ predicted & Reisner et al. (1998) $V_{\text {fall }}=700 \times 2 r_{\text {ice }}$ \\
\hline $\mathrm{D}$ & Simple ice, Dudhia (1989)—No prediction of $\mathrm{N}_{\text {ice }}$ & $\begin{array}{l}\text { Heymsfield and Donner }(1990): V_{\text {fall }}=a\left(q_{\text {ice }} \rho_{\text {air }}\right)^{b} \\
\quad \mathrm{a}=3.29 \text { and } b=0.16\end{array}$ \\
\hline
\end{tabular}


prognostic variables are the mass content of precipitation water and the cloud water. The distinction between liquid and solid water is made by assuming a temperature threshold $T_{t}\left({ }^{\circ} \mathrm{C}\right)$ as a separation criterion between liquid water (and rain) and ice (and snow). Three threshold values of $T_{t}\left(0^{\circ},-10^{\circ},-20^{\circ} \mathrm{C}\right)$ have been tested for the conversion of ice/liquid water, and the one giving the best results $\left(T_{t}=-20^{\circ} \mathrm{C}\right)$ is used hereafter. The initial threshold value of $T_{t}=0^{\circ} \mathrm{C}$ is kept for the conversion of snow/rain. Furthermore, there is no prognostic ice number concentration for this scheme.

The terminal velocity is a key parameter in microphysics schemes. In theory it is a function of the particle size, which is the case in the Reisner et al. (1998) original scheme. However it is applied to a mean particle size, which makes the terminal velocity much smaller than that of the most massive particles accounting for most of the ice content sedimentation mass loss. In most simulated clouds this terminal velocity was found to be about an order of magnitude smaller than that obtained using the Heymsfield and Donner (1990) formula, which is derived from observed precipitation rates. This was suspected to be one of the reasons why the Reisner et al. (1998) original scheme overproduces ice clouds, as we shall see for scheme denoted as C. Furthermore, the terminal velocity is known as a parameter with large uncertainty; it is then interesting to test it. In scheme A, $V_{t}$ is taken as a variable function of the simulated ice mixing ratio $q_{\text {ice }}\left(\mathrm{kg} \mathrm{kg}^{-1}\right)$, the density of air $\rho_{\text {air }}\left(\mathrm{kg} \mathrm{m}^{-3}\right)$ following Zurovac-Jevtic and Zhang (2003). Scheme B is similar to A, but uses the Heymsfield and Donner (1990) parameterization.

\section{Observations}

\section{a. Instruments}

The SIRTA ground-based site $\left(48.7^{\circ} \mathrm{N}, 2.2^{\circ} \mathrm{E}\right.$; http:// sirta.lmd.polytechnique.fr) provides routine observations of the atmospheric column (Haeffelin et al. 2004) collected with the following active and passive remote sensing instruments.

\section{1) A GROUND-BASED 532-NM LIDAR}

The SIRTA 532-nm lidar operates 4 days a week from 8 A.M. to 8 P.M. except when it is raining. The nominal temporal resolution is $10 \mathrm{~s}$, and the vertical resolution is $15 \mathrm{~m}$. It is a zenith-viewing lidar that measures both the backscattered signal and linear depolarization ratio. The lidar signal is normalized to the lidar signal that would be measured in a free-particle area, that is, when there are only molecules. It is deduced from Météo-France radiosonde pressure and tempera- ture profiles (which are launched every day in Trappes, $15 \mathrm{~km}$ away from SIRTA). With typical value of wind at cirrus cloud altitude $\left(10 \mathrm{~m} \mathrm{~s}^{-1}\right)$, the typical MM5 mesh residence time is about $10 \mathrm{~min}$; therefore lidar profiles are averaged over $10 \mathrm{~min}$ and taken every hour for comparison with MM5 outputs. In this study, the water phase is deduced from the lidar depolarization ratio, which is the ratio of the perpendicular to the parallel backscattered lidar signals (Sassen 1991; Noel et al. 2001). The depolarization ratio is normalized to $2.8 \%$ in a molecular portion of the atmosphere. In presence of clouds, ratios lower than 5\% are associated with liquid water (spherical particles), and ratios larger than $20 \%$ with ice and/or snow (nonspherical particles). When ratio ranges between $5 \%$ and $20 \%$, the cloud is classified as having a mixed phase.

\section{2) A Ground-BAsed 95-GHz RADAR}

The SIRTA 95-GHz radar has operated continuously since October 2002. Data are acquired with a 1-s temporal resolution, and vertical resolution is $60 \mathrm{~m}$. The reflectivity and Doppler velocity profiles are obtained from 0.2 to $15 \mathrm{~km}$. The sensitivity of the radar is around $-50 \mathrm{dBZ}$ at $1 \mathrm{~km}$ for the period considered in the study, with a $20 \times \log \left(z_{\mathrm{km}}\right)$ sensitivity loss every kilometer. For the same reason as for the lidar, radar profiles are averaged over $10 \mathrm{~min}$ and sampled every hour.

\section{3) Radiative Flux STATiON}

The pyrheliometer measures the direct shortwave flux $\mathrm{SW}_{\mathrm{dct}}\left(\mathrm{W} \mathrm{m}^{-2}\right)$ and the pyranometer measures the diffuse shortwave flux $\mathrm{SW}_{\mathrm{dff}}\left(\mathrm{W} \mathrm{m}^{-2}\right)$. The total downward shortwave flux SW $\left(\mathrm{W} \mathrm{m}^{-2}\right)$ is simply $\mathrm{SW}=$ $\mathrm{SW}_{\text {diff }}+\cos \theta \mathrm{SW}_{\mathrm{dct}}$, where $\theta$ is the solar zenith angle. The pyrgeometer measures the net longwave flux $\mathrm{LW}_{n}$ $\left(\mathrm{W} \mathrm{m}^{-2}\right)$. The downward longwave flux is $\mathrm{LW}=$ $\mathrm{LW}_{n}+\sigma T_{i}^{4}$, where $\sigma$ is the Stefan constant $(5.67 \times$ $\left.10^{-8} \mathrm{SI}\right)$ and $T_{i}$ is the instrument temperature (K). The fluxes are averaged over $10 \mathrm{~min}$ and sampled every hour.

\section{b. Selected cases}

Sixty-two cloudy days have been subjectively selected based on lidar and radar observations. Days with observations corresponding to low-level liquid clouds only or clear sky only were systematically removed. The clouds are separated into three classes that have different geophysical properties (altitude and temperature, cloud particle microphysical properties, radiative impact): 
a. only lidar

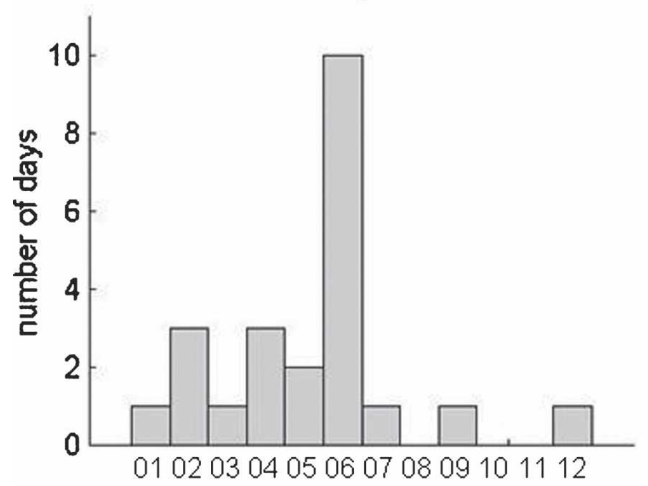

c. only radar, WE

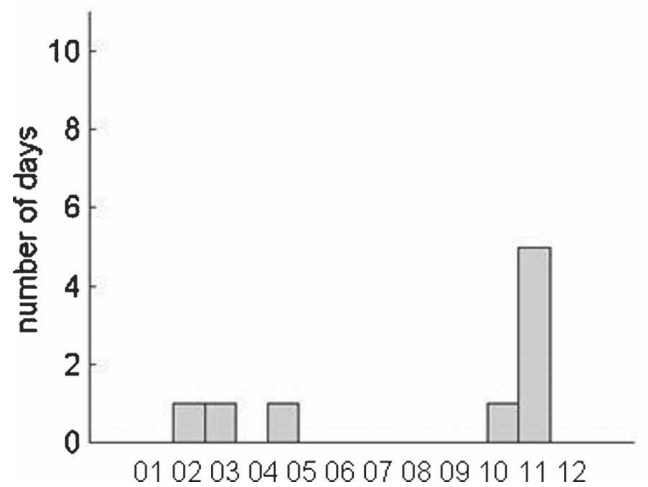

b. only radar

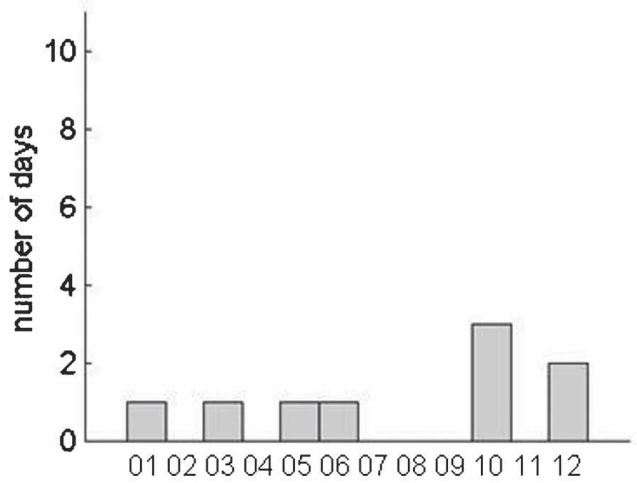

d. lidar and radar

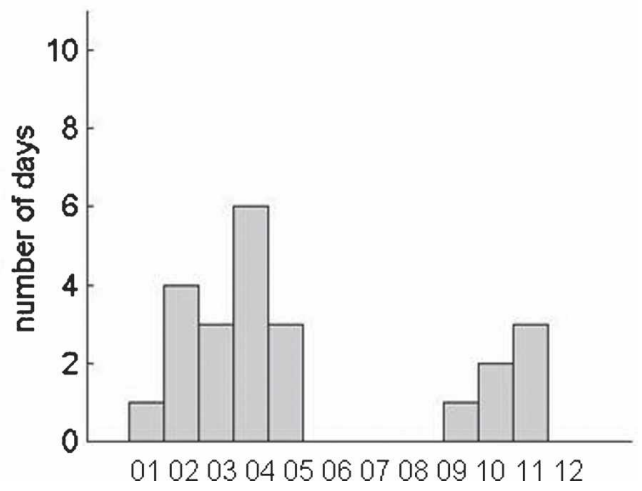

FIG. 2. Number of cases per month for 2003 and 2004. Cases of (a) only lidar measurements, (b) only radar measurements because of meteorological condition, (c) only radar measurements because of lidar maintenance, and (d) both lidar and radar measurements.

(i) In 21 cases, there are only lidar measurements because clouds are too thin to be detected by radar. Those clouds are classified as "thin" with optical depth smaller than 1.

(ii) In 23 cases, both lidar and radar display a cloudy signal, and the two measurements were simultaneously available. Those clouds are classified as "medium" with optical depth larger than 1 , and include cases for which the lidar penetrates only a portion of the cloud.

(iii) In 18 cases, only the radar was available: in 9 cases, because meteorological conditions did not allow lidar measurements (rain or cloud too thick), the clouds are classified as "thick"; in 9 cases because lidar was off (weekend or maintenance), the clouds could either be medium or thick clouds.

When lidar observations are available, ice/snow cloud cases are selected based on lidar depolarization profiles containing values larger than $10 \%$ (i.e., presence of nonspherical crystals; Sassen 1991; Noel et al. 2001).
When only radar observations are available, a cloud case is selected only when a high-altitude cloud layer (typically $8-10 \mathrm{~km}$ ) is present during the day, even if it does not persist all day long. These clouds may contain ice, snow, and water.

Figure 2 summarizes the 62 different days: 32 cases from February to December 2003 and 30 cases from January to June 2004. The maximum of thin cloud occurrence is in May, and the maximum of medium cloud occurrence is in April.

\section{The ACTSIM model-to-observation comparison method}

To compare model and observations, shortwave and longwave downward fluxes are computed directly from model outputs. Comparisons of lidar, radar profiles, and vertical velocity require a postprocessing of model outputs. We developed a program [active remote sensing simulator (ACTSIM)] that allows the simulation of lidar and radar signals from modeling variables. 
TABLE 2. Differences between the four schemes concerning ACTSIM calculation; $\rho_{\text {liq/ice/snow }}$ is the simulated density of liquid water/ice/snow $\left(1000 \mathrm{~kg} \mathrm{~m}^{-3} / 500 \mathrm{~kg} \mathrm{~m}^{-3} / 100 \mathrm{~kg} \mathrm{~m}^{-3}\right)$ for the $\mathrm{A}, \mathrm{B}$, and $\mathrm{C}$ schemes.

\begin{tabular}{|c|c|c|}
\hline & $\mathrm{A}, \mathrm{B}$, and $\mathrm{C}$ & $\mathrm{D}$ \\
\hline$N_{\text {liq }}\left(\mathrm{m}^{-3}\right)$ & Fixed value (in the scheme) $1 \times 10^{8} \mathrm{~m}^{-3}$ & $\left(3 q_{\text {liq }} / \rho_{\text {air }}\right) /\left(4 \pi r_{\text {liq }}^{3} \rho_{\text {liq }}\right)$ \\
\hline$N_{\text {ice }}\left(\mathrm{m}^{-3}\right)$ & Prognostic & $\left(3 q_{\text {ice }} / \rho_{\text {air }}\right) /\left(4 \pi r_{\text {ice }}^{3} \rho_{\text {ice }}\right)$ \\
\hline$N_{\text {snow }}\left(\mathrm{m}^{-3}\right)$ & $\begin{array}{l}N_{0, \text { snow }}^{3 / 4}\left(\rho_{\text {air }} q_{\text {snow }} / \rho_{\text {snow }} \pi\right)^{1 / 4} N_{0, \text { snow }} \text { variable in } \\
\text { the scheme }\end{array}$ & $\begin{array}{l}N_{0, \text { snow }}^{3 / 4}\left(\rho_{\text {air }} q_{\text {snow }} / \rho_{\text {snow }} \pi\right)^{1 / 4} N_{0, \text { snow }} \text { fixed in the scheme to } \\
2 \times 10^{7} \mathrm{~m}^{-4}\end{array}$ \\
\hline$r_{\text {liq }}(\mathrm{m})$ & $\left(3 q_{\mathrm{liq}} \rho_{\mathrm{air}} / N_{\mathrm{liq}} 4 \pi \rho_{\mathrm{liq}}\right)^{1 / 3}$ & Fixed value (in ACTSIM): $10 \times 10^{-6} \mathrm{~m}$ \\
\hline$r_{\text {ice }}(\mathrm{m})$ & $\left(3 q_{\text {ice }} \rho_{\text {air }} / N_{\text {ice }} 4 \pi \rho_{\text {ice }}\right)^{1 / 3}$ & Fixed value (in ACTSIM): $30 \times 10^{-6} \mathrm{~m}$ \\
\hline$r_{\text {snow }}(\mathrm{m})$ & $\left(3 q_{\text {snow }} \rho_{\text {air }} / N_{\text {snow }} 4 \pi \rho_{\text {snow }}\right)^{1 / 3}$ & $\left(3 q_{\text {snow }} \rho_{\text {air }} / N_{\text {snow }} 4 \pi \rho_{\text {snow }}\right)^{1 / 3}$ \\
\hline
\end{tabular}

\section{a. Particle size and distribution}

For $\mathrm{A}, \mathrm{B}$, and $\mathrm{C}$ schemes, ice number concentration is available and the snow and ice particle radii $r_{\text {snow/ice }}$ (radius of a particle of mean mass, in meters) are deduced from the microphysical scheme (Table 2) using the ice/snow particle numbers $N_{\text {ice/snow }}\left(\mathrm{m}^{-3}\right)$ and their respective mixing ratio $q_{\text {ice/snow }}\left(\mathrm{kg} \mathrm{kg}^{-1}\right) ; N_{\text {ice }}$ is a direct prognostic model variable while $N_{\text {snow }}$ can be deduced from the snow mixing ratio $q_{\text {snow }}$, which is also a prognostic variable and from the intercept slope parameter $N_{0, \text { snow }}\left(\mathrm{m}^{-4}\right)$ that is variable (formulas in Reisner et al. 1998).

For scheme D, $r_{\text {ice }}$ could also be derived from $N_{\text {ice }}$ and $q_{\text {ice }}$ as $N_{\text {ice }}$ could be recalculated as a function of the temperature [using a Fletcher curve, following Fletcher (1962)]. Tests have been done using this approach showing that it leads to unphysical particle sizes (typically $2.7 \times 10^{-2} \mu \mathrm{m}$ ). As the ice particle size in this scheme determines only the microphysical rates, such as deposition, that become faster than the model time step for small particles, and so insensitive to the particle size, $r_{\text {ice }}$ has been kept constant at $35 \mu \mathrm{m}$ (10, 50, and $100 \mu \mathrm{m}$ have also been tested); $N_{\text {ice }}$ is then simply derived from $r_{\text {ice }}$ and $q_{\text {ice, }}$ and $r_{\text {snow/ice }}$ is calculated in the same manner as for schemes A, B, and C, but using a constant value of $N_{0, \text { snow }}\left(2 \times 10^{7} \mathrm{~m}^{-4}\right)$ (Table 2).

In the $\mathrm{A}, \mathrm{B}$, and $\mathrm{C}$ schemes, the liquid water particle concentration $N_{\text {liquid }}\left(\mathrm{m}^{-3}\right)$ is considered to be constant $\left(1 \times 10^{8} \mathrm{~m}^{-3}\right)$, which allows one to derive the liquid particle effective radius $r_{\text {liquid }}(\mathrm{m})$ from $N_{\text {liquid }}$ and $q_{\text {liquid }}$ (prognostic variable in $\mathrm{kg} \mathrm{kg}^{-1}$ ). The $\mathrm{D}$ scheme is in part based on the Kessler (1969) scheme, which does not make any assumption about the liquid particle size and concentration. Hence, for this scheme, $r_{\text {liquid }}$ is supposed to be constant in ACTSIM and equal to $10 \mu \mathrm{m}$ $\left(6,20\right.$, and $50 \mu \mathrm{m}$ have also been tried); $N_{\text {liquid }}$ is then derived from $r_{\text {liquid }}$ and $q_{\text {liquid. }}$.

To simulate lidar and radar profiles as realistically as possible, we do not consider a single value of particle size, but a size distribution in ACTSIM. For ice and liquid water, we use a lognormal distribution with a modal radius equal to $r_{\text {ice/liquid: }}$

$n_{\text {ice/liquid }}(\Re) d \Re=\frac{N_{\text {ice/liquid }}}{\sqrt{2 \pi} \Re \ln \sigma} e^{-\left[\ln ^{2}\left(\Re / r_{\text {ice/liquid }} / 2 \ln ^{2} \sigma\right]\right.} d \Re$,

where $n=n_{\text {ice/liquid }}(\Re) d \Re\left(\mathrm{m}^{-4}\right)$ is the number of particles of ice/liquid that have a radius between $\Re$ and $\Re$ $+d \Re(\mathrm{m})$. The $\sigma$ value is constant and equal to 1.2 for ice clouds (Heymsfield and Platt 1984). The values of $\Re$ vary between $\Re_{\text {min }}=0.27 \times r_{\text {ice/liquid }}$ and $\Re_{\text {max }}=3.44 \times$ $r_{\text {ice/liquid }}\left(\Re_{\min }=10.7 \mu \mathrm{m}\right.$ and $\Re_{\max }=139.5 \mu \mathrm{m}$ for $\left.r_{\text {ice }}=40 \mu \mathrm{m}\right)$.

The shape of the snow size distribution is taken from the microphysical schemes as a Marshall-Palmer (1948) size distribution:

$$
n_{\text {snow }}(\Re) d \Re=N_{0, \text { snow }} e^{-(\Re / 2)\left(\pi \rho_{\text {snow }} N_{O, \text { snow }} / \text { pair }_{\text {ar }} q_{\text {snow }}\right)^{1 / 4}} d \mathfrak{R},
$$

where $n_{\text {snow }}(\Re) d \Re\left(\mathrm{m}^{-4}\right)$ is the number of particles of snow that have a radius between $\Re$ and $\Re+d \Re$.

\section{b. Lidar profile simulation}

The 532-nm lidar equation giving the backscattered signal power at a level $z$ is

$P(z) z^{2}=\left[\beta_{\mathrm{mol}}(z)+\beta_{\mathrm{par}}(z)\right] e^{-2 \int_{0}^{z}\left[\eta \alpha_{\mathrm{par}}(z)+\alpha_{\mathrm{mol}}(z)\right] d z}$,

where $P(z) z^{2}$ is the simulated lidar backscattered normalized signal (i.e., corrected of the calibration constant) at the altitude $z$, expressed in $\mathrm{m}^{-1} \mathrm{sr}^{-1}$.

The simulated particle attenuation by scattering $\alpha_{\mathrm{par}}$ $\left(\mathrm{m}^{-1}\right)$ is the sum of the contribution of snow $\alpha_{\text {snow }}$ $\left(\mathrm{m}^{-1}\right)$, liquid water $\alpha_{\text {liquid }}\left(\mathrm{m}^{-1}\right)$, and ice water $\alpha_{\text {ice }}$ $\left(\mathrm{m}^{-1}\right)$ :

$$
\alpha_{\text {par }}=\alpha_{\text {snow }}+\alpha_{\text {liquid }}+\alpha_{\text {ice }}
$$

and

$$
\alpha_{\text {snow/iquid/ice }}=\pi r_{\text {snow/liquid/ice }}^{2} Q N_{\text {snow/liquid/ice }},
$$


where $Q$ is the particle scattering efficiency that is equal to 2 at $532 \mathrm{~nm}$ because particles of snow, ice, and liquid water are much larger than the lidar wavelength (Ulaby et al. 1943). Furthermore, the multiple scattering correction parameter $[\eta$ in Eq. (3)] is taken equal to 0.5 (Platt 1973). The exponential term in (3) represents the optical depth between levels 0 and $z$, and contains only scattering terms as there is no absorption by cloud particles at $532 \mathrm{~nm}$.

The simulated lidar backscattering coefficient by particles, $\beta_{\mathrm{par}}\left(\mathrm{m}^{-1} \mathrm{sr}^{-1}\right)$, corresponds to the sum of the contribution of snow $\beta_{\text {snow }}\left(\mathrm{m}^{-1} \mathrm{sr}^{-1}\right)$, ice $\beta_{\text {ice }}$ $\left(\mathrm{m}^{-1} \mathrm{sr}^{-1}\right)$, and liquid water $\beta_{\text {liquid }}\left(\mathrm{m}^{-1} \mathrm{sr}^{-1}\right)$ :

$$
\beta_{\text {par }}=\beta_{\text {snow }}+\beta_{\text {ice }}+\beta_{\text {liquid }},
$$

with

$$
\beta_{\text {snow/ice/liquid }}=\frac{P_{\pi, \text { snow/ice/liquid }}}{4 \pi} \alpha_{\text {snow/ice/liquid }},
$$

where $P_{\pi, \text { snow/ice/liquid }}$ is the simulated scattering phase function in backscattering. Particles are assumed to be spherical in the four microphysical schemes; $P_{\pi \text {,snow/ice/liquid }}$ is computed with Mie theory for the size distributions given in (1) and (2) in order to be consistent with the model assumptions.

The lidar backscattering coefficient by molecules is $\beta_{\mathrm{mol}}\left(\mathrm{m}^{-1} \mathrm{sr}^{-1}\right)$. It depends on temperature $T(\mathrm{~K})$ and pressure $p(\mathrm{hPa})$ profiles (Collis and Russel 1976):

$$
\beta_{\mathrm{mol}}=\frac{p}{K T}\left(5.45 \times 10^{-32}\right)\left(\frac{\lambda}{0.55}\right)^{-4.09}
$$

and

$$
\alpha_{\mathrm{mol}}=\frac{\beta_{\mathrm{mol}}}{0.119},
$$

where $K$ is the Boltzmann constant $\left(1.38 \times 10^{-23} \mathrm{~J} \mathrm{~K}^{-1}\right)$, and $\lambda$ is the wavelength; $T$ and $p$ are given by the radiosonde as for the measured lidar profile normalization.

The comparison between observed and simulated lidar signals is achieved by renormalizing the observed lidar signal on the simulated one in a particle free area (i.e., molecules only) just under the cloud, so that the two profiles can be compared within the cloud.

\section{c. Radar profile simulation}

The radar reflectivity in $\mathrm{dB}$ is given by

$$
\mathrm{dB} z=10 \log \left(Z_{\text {snow }}+Z_{\text {ice }}+Z_{\text {liquid }}\right) \text {, }
$$

where $\mathrm{dB} Z$ values inferior to $-50+20 \log (Z)$ are eliminated because of the radar sensitivity; $Z_{\text {snow/ice/liquid }}$ $\left(\mathrm{m}^{3}\right)$ is the contribution of snow/ice/liquid to the simulated radar reflectivity:

$$
\begin{aligned}
Z_{\text {snow/ice/liquid }}= & 10^{18}\left(\frac{K_{\text {snow/ice/liquid }}^{2}}{K_{\text {liquid }}^{2}}\right)\left(\frac{1}{0.917}\right)^{2} \\
& \int_{D_{\text {eq }, \min }}^{D_{\text {eq,max }}} D_{\text {eq }}^{6} n_{\text {snow/ice/liquid }}\left(D_{\text {eq }}\right) d D_{\text {eq }} .
\end{aligned}
$$

The dielectric factor $\left(K_{\text {snow/ice/liquid }}^{2}\right)$ at $94 \mathrm{GHz}$ is equal to 0.176 for ice and snow and 0.930 for liquid water; $D_{\text {eq }}$ $(\mathrm{m})$ is the equivalent diameter:

$$
\begin{aligned}
D_{\text {eq,snow/ice/liquid }}= & \left(\frac{\rho_{\text {snow/ice/liquid }}}{\rho_{\text {liquid }}}\right)^{(1 / 3)} \\
& \times 2 \Re_{\text {snow/ice/liquid. }}
\end{aligned}
$$

The radar reflectivity is calculated using the size distributions described in section 4a. The liquid water attenuation is not considered, as most of the selected clouds are ice or mixed clouds.

\section{d. Doppler velocity simulation}

The Doppler velocity $V\left(\mathrm{~m} \mathrm{~s}^{-1}\right)$, measured by the vertically pointing radar, is the reflectivity weighted mean radial velocity of the particles, that is, the sum of the vertical velocity of air $w$ (prognostic variable in $\mathrm{m} \mathrm{s}^{-1}$ ) and the reflectivity weighted terminal velocity $v_{t}$ of the ice particles $\left(\mathrm{m} \mathrm{s}^{-1}\right)$ :

$$
V=v_{t}+w
$$

where

$$
v_{t}=\frac{\int_{D_{\text {eq,min }}}^{D_{\text {eq,max }}} n_{\text {ice }}\left(D_{\text {eq,ice }}\right) u_{t, \text { ice }}\left(D_{\text {eq,ice }}\right) D_{\text {eq,ice }}^{6} d D_{\text {eq,ice }}+\int_{D_{\text {eq,min }}}^{D_{\text {eq,max }}} n_{\text {snow }}\left(D_{\text {eq, snow }}\right) u_{t, \text { snow }}\left(D_{e q, \text { snow }}\right) D_{\text {eq,snow }}^{6} d D_{\text {eq,snow }}}{\int_{D_{\text {eq,min }}}^{D_{\text {eq,max }}} n_{\text {ice }}\left(D_{\text {eq,ice }}\right) D_{\text {eq,ice }}^{6} d D_{\text {eq,ice }}+\int_{D_{\text {eq,min }}}^{D_{\text {snow }}}\left(D_{\text {eq,snow }}\right) D_{\text {eq,snow }}^{6} d D_{\text {eq,snow }}} .
$$



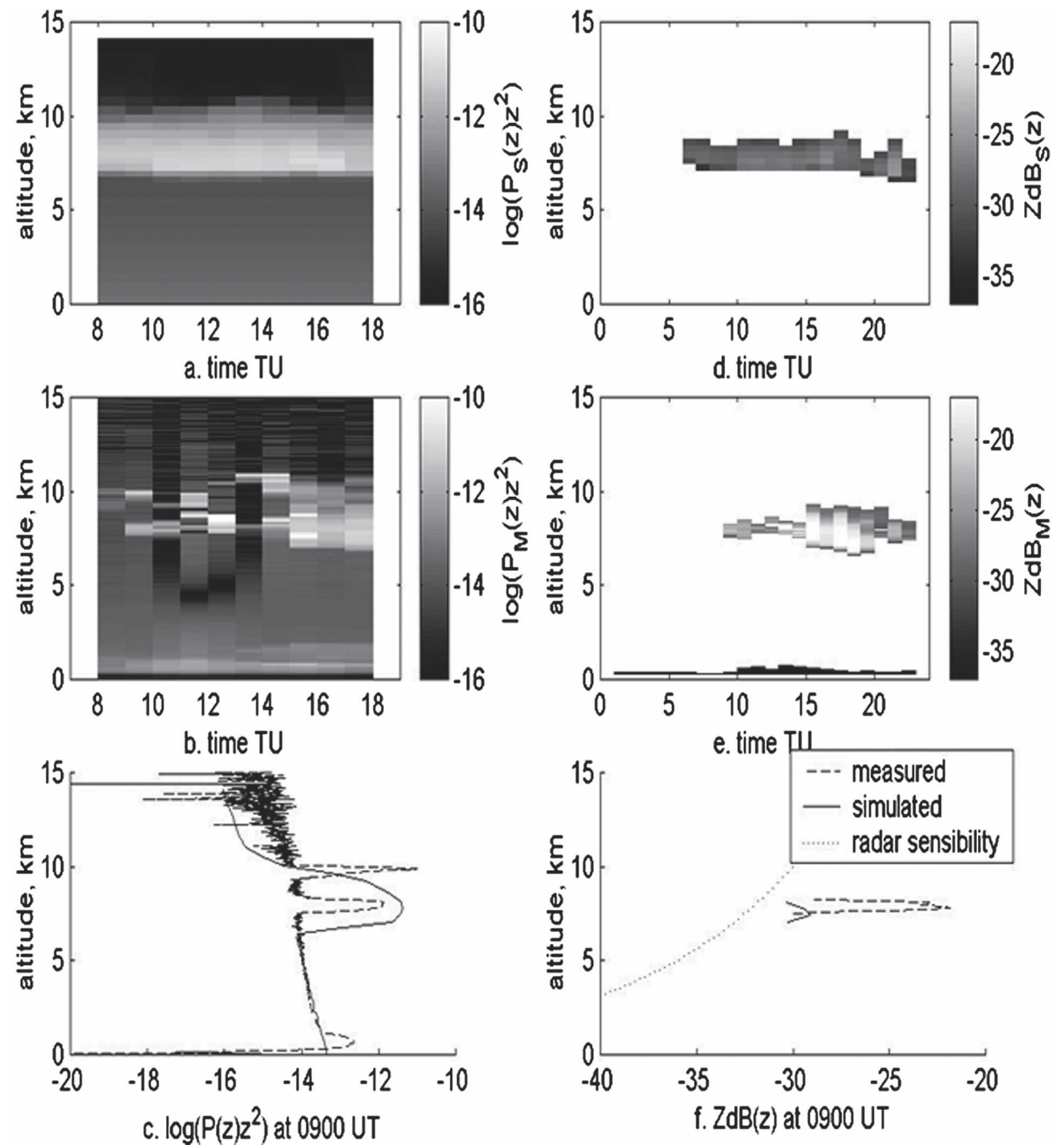

FIG. 3. B scheme for 17 Oct 2003: (a) time evolution of logarithm of simulated lidar backscattered profile, (b) same as (a) but for measured profile, (c) logarithm of simulated and measured lidar profile at 0900 UTC, (d) same as (a) but for simulated radar reflectivity, (e) same as (a) but for measured radar reflectivity, and (f) simulated and measured radar profiles at 0900 UTC, where simulation is solid line, measurement is dotted line, and radar sensitivity is dashed line.

The monocrystal terminal fall velocities $u_{t, \text { ice/snow }}\left(D_{\text {eq }}\right)$ $\left(\mathrm{m} \mathrm{s}^{-1}\right)$ are a function of $D_{\text {eq.ice/snow. The expressions of }}$ $u_{t, \text { ice/snow }}\left(D_{\text {eq }}\right)$ are specific to each microphysical scheme (Heymsfield and Donner 1990; Zurovac-Jevtic and Zhang 2003; Reisner et al. 1998). These are those expressions (mostly based on in situ measurements) that are used to build the expression of $V_{\text {fall }}$ given in Table 1. Liquid water (cloud water and rain) and graupel are not considered in (14) as they are almost inexistent in the selected cases.
In the following sections, all variables related to simulation will be indexed with "S," and all variables related to measurements with "M."

\section{Illustrative example: 17 October 2003}

The date 17 October 2003 illustrates the complementarities of lidar/radar/fluxes. The lidar and radar observed and simulated (using scheme B) signals on 17 October 2003 are shown in Fig. 3. For both simulations 
and measurements, the ice cloud is already present when the lidar measurements start at 0800 UTC. The cloud lies at an altitude of about $10 \mathrm{~km}$ at the beginning of the day in observations and between 6 and $10 \mathrm{~km}$ for simulations throughout the day. The observed cloud evolves during the day and its altitude is the same as for simulations at the end of the day. The observations show a boundary layer below $1 \mathrm{~km}$, which is not reproduced in simulations because prognostic aerosols are not taken into account in MM5. Nevertheless, it does not influence the values of lidar signal within the cloud as simulated and measured lidar profiles are both normalized to the same value just below the cloud as shown in Fig. 3c.

Figure $3 \mathrm{~d}$ is the time evolution of simulated radar reflectivity from 0000 to 2300 UTC. The simulated cloud appears at $0500 \mathrm{UTC}, 4 \mathrm{~h}$ before the observed one. Between 0800 and 0900 UTC the cloud is too thin to be detected by the radar whereas it is already present in the simulated radar signal (Fig. 3d). Nevertheless, Fig. 3f shows that the simulated reflectivity is smaller than radar sensitivity. After 0900 UTC, the cloud is present in both measurements and simulations. Nevertheless, the cloud height is larger than 1 to $2 \mathrm{~km}$ in simulations than in measurements. As for the lidar, there is a small boundary layer detected by radar that is not represented by the simulated reflectivity. The observed cloud has a much more complex structure than the simulated cloud. It could be due to coarse vertical and horizontal model resolutions, but even at resolution of 5 or $15 \mathrm{~km}$, there is a substantial amount of subgrid variability in the horizontal, and a lower horizontal resolution does not immediately imply simulations of worse quality. The observed cloud structure displays multiple layers (Fig. 3c) that are not reproduced by the model. These figures therefore show that gross features of the thin ice clouds can be represented in mesoscale models.

Figures $4 \mathrm{a}$ and $4 \mathrm{~b}$ show the time evolution of the cloud thermodynamical phase for simulations and observations, measured from the method described in section 3. The thermodynamical phases are compared only within the cloud [cloud base and top are derived using the Morille et al. (2005, manuscript submitted to J. Atmos. Oceanic Technol., hereafter STRAT) algorithm]. Figure 4 shows that the observed cloud is mostly composed of mixed phase with a few ice/snow pixels, whereas the simulated cloud is composed exclusively of snow and ice. This is not due to the temperature within the cloud because the altitude of the cloud (and so its temperature) is well represented by the model. It could be due to the microphysical processes such as deposition or conversion of ice to snow.
Figure 5 shows the temporal evolution of Doppler velocity for simulations (Fig. 5a) and measurements (Fig. 5b). Negative values correspond to downward motions. The order of magnitude is similar, but the measured absolute values are about $0.4 \mathrm{~m} \mathrm{~s}^{-1}$ larger than the simulated ones. The measured Doppler velocity is the contribution of both $V_{t}$ and $w$, but the difference between simulations and observations is only due to the contribution of $V_{t}$ because the range of w is very weak (typically a few $\mathrm{cm} \mathrm{s}^{-1}$ ). The fact that the simulated particles fall more slowly than the real ones is consistent with the lidar/radar information: a cloud too persistent and with a too large height.

Figure 6 shows downward longwave flux (LWF) and shortwave flux (SWF) time evolution for both simulations and measurements. The simulated SWF follows a bell shape, which is consistent with the rather homogeneous cloud shown in Figs. 3a and 3d. Despite the difference in simulated/observed cloud structures, SWFs are in fair agreement. That means that despite the fact that the cloud has a too large height, its optical depth is well represented. Hence this overestimation of the cloud extent does not have a large impact on SWFs, which is an important variable. Nevertheless, in cloudfree conditions (before 0500 UTC) the simulated LWF is $40 \mathrm{~W} \mathrm{~m}^{-2}$ lower than the measured one, which strongly suggests that the clear-sky LWF is not well computed in the model. Hence, in presence of clouds, the differences or similarities between simulated and measured LWF cannot be interpreted correctly.

\section{Statistical analysis}

The systematic character of the model/observation comparisons are now evaluated through a statistical analysis carried out over the 62 available days. The comparison is actually made on hourly sampled data, observations being in fact 10-min averages taken every hour.

\section{a. Cloud occurrence}

The STRAT algorithm is applied for both observations and simulations, and hits and misses are counted for each microphysical option. Figure 7a shows the rates of cloud hits, nondetection, and false alarm. For the four schemes, simulated and measured clouds match in more than $65 \%$ of cases, and this agreement is maximal for scheme D (the "simple ice" scheme), with a hit rate of about $75 \%$. All schemes overestimate the cloud occurrence in about $20 \%$ of cases $[25 \%$ for scheme C, the original Reisner et al. (1998) scheme]. Scheme B [Reisner et al. (1998) with the Heymsfield 

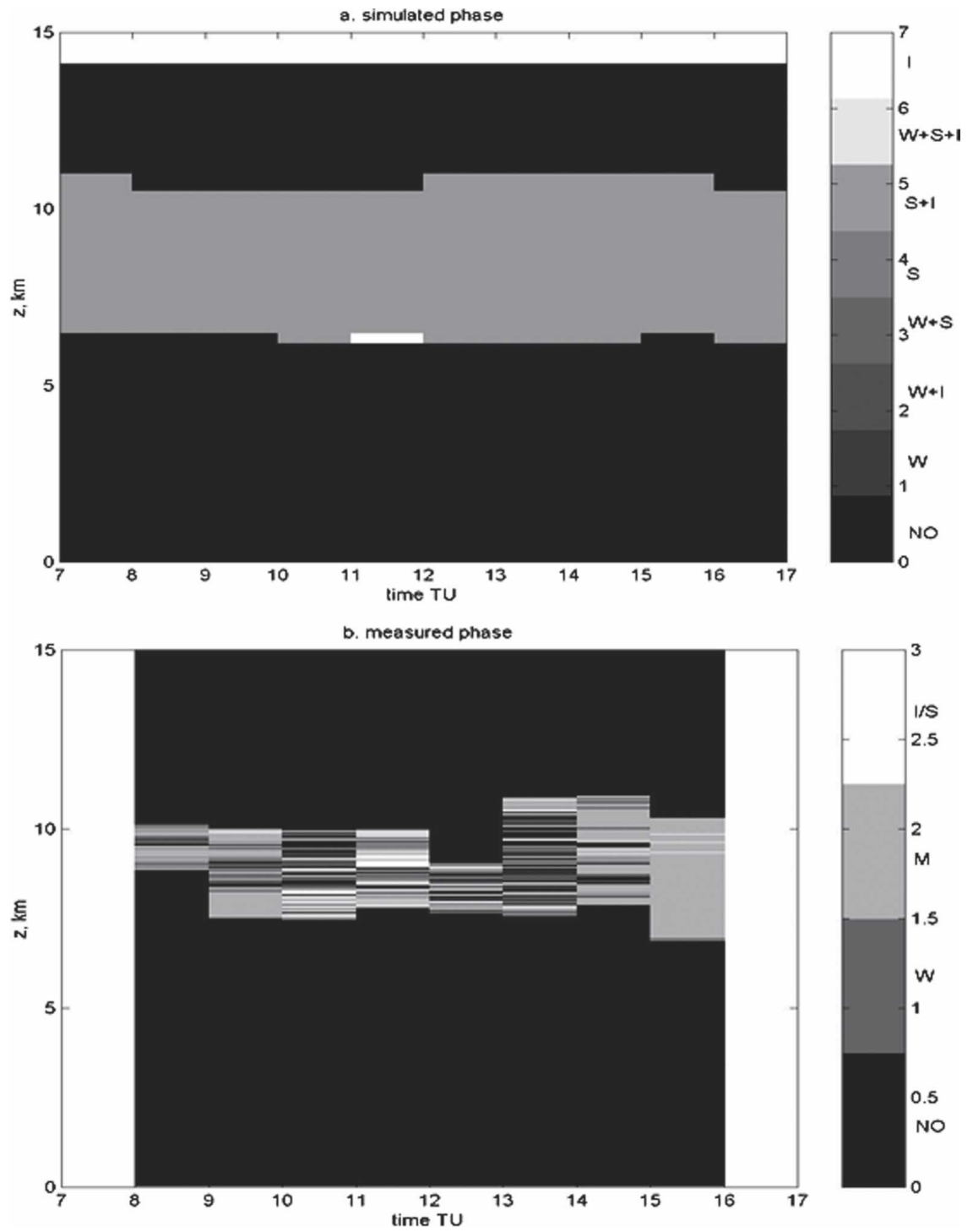

FIG. 4. B scheme for 17 Oct 2003: (a) time evolution of simulated phase; (b) same as (a) but retrieved from lidar depolarization ratio measurements. Here, NO means no cloud, W means liquid water, S means snow, and I means ice.

and Donner (1990) terminal velocity] is the most balanced scheme, with as many false alarms as misses.

The balance between hits and misses may, however, be biased since our case selection is based on observations only. The number of false alarms is probably underestimated since there may be unselected cases with a model cloud and no actual cloud during other days. Therefore, a general conclusion is that these microphysics schemes tend to produce too many ice clouds. This result is almost equivalent for the four different microphysical schemes; hence the differences between simulations and observations must be due to something other than the microphysical parameterizations. As was mentioned in section 2a, it could be due to the parameterizations of subgrid-scale turbulence and convection processes, which are not forced by the nudging procedure though they play a fundamental role in cloud simulations.

\section{b. Cloud lifetime}

To study the lifetime (hours) of clouds, 15 days (among 62) corresponding to persistent homogeneous cloud layers where beginning and end of cloud is observed have been subjectively selected. Figure $7 \mathrm{~b}$ shows the probability density function (PDF) of the difference between the simulated and the measured cloud life- 
a. simulated radar doppler velocity

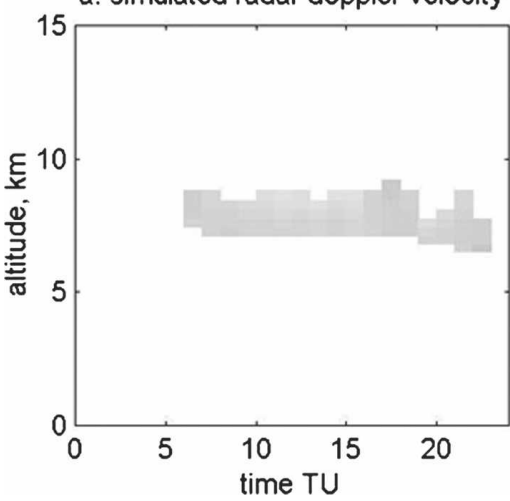

b. measured radar doppler velocity

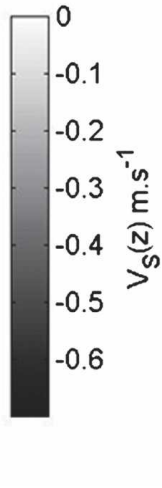

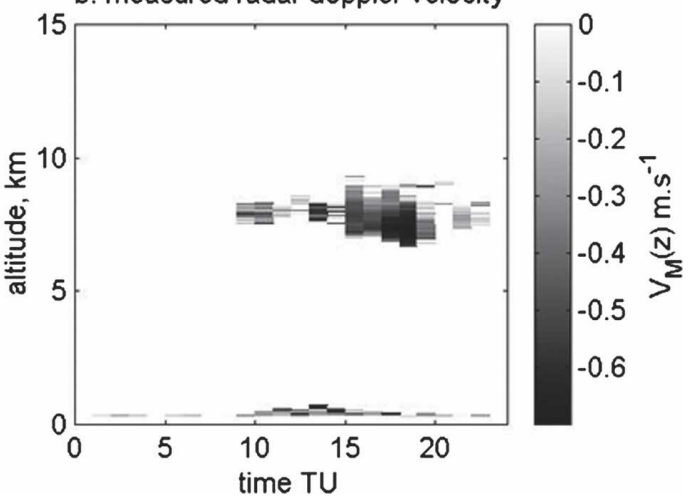

FIG. 5. B scheme for 17 Oct 2003: (a) time evolution of simulated vertical velocity; (b) same as (a) but for measured vertical velocity.

time. In the four schemes, the tendency is to simulate longer lifetime than the observed one, and the maximal difference is more than $6 \mathrm{~h}$, which is found in $20 \%$ of cases whatever the scheme. For schemes $\mathrm{C}$ and $\mathrm{D}$, the difference between the simulated and the measured cloud lifetime is less than $1 \mathrm{~h}$ for $50 \%$ of cases and less than $45 \%$ for scheme A. For all schemes, in $15 \%-20 \%$ of cases the measured cloud is 3 to $5 \mathrm{~h}$ more persistent than the simulated one. Scheme B exhibits the most balanced results in terms of cloud lifetime. Despite those differences, the general tendency is the same for all microphysical schemes, so this tendency must be due to something that is equivalent for all microphysical schemes, such as the parameterizations of subgrid thermodynamical processes that have a strong impact on the creation of the conditions for the existence of clouds. However the small size of the sample here does not allow intercomparing schemes in a statistically significant manner.

\section{c. Cloud thermodynamical phase}

For all the cases with available lidar measurements, simulated and measured thermodynamical phases are determined and compared at every hour and altitude, following the same method as for the illustrative case (section 5). Figure 8a shows the simulated cloud phase when the observed cloud is liquid water (i.e., $20 \%$ of the total lidar dataset): scheme $\mathrm{D}$ with the $-20^{\circ}$ threshold gives the best phase estimation with $60 \%$ of liquid water, whereas the three other schemes produce ice and snow instead of liquid water in more than $80 \%$ of cases. Figure $8 \mathrm{~b}$ corresponds to the observed mixedphase cloud (i.e., $40 \%$ of the total lidar dataset). The four schemes are not consistent with observation as they do not lead to a mix of liquid water and ice and/or snow. When the lidar depolarization ratio leads to ice and/or snow crystals (Fig. 8c), the simulated phase is a snow and/or ice for almost $100 \%$ of cases for A, B, and C schemes and $80 \%$ for the D scheme. These results show that the Reisner et al. (1998) scheme generally overestimates the fraction of ice/snow in high clouds. An underestimate of supercooled water in schemes $\mathrm{A} / \mathrm{B} / \mathrm{C}$ indicates either that the model is not capturing the strength of the vertical upward motion well in regions where supercooled water is produced, or that there is too much ice nucleation at these warmer temperatures. Scheme D only succeeds because of the $-20^{\circ} \mathrm{C}$ threshold, otherwise it also would not produce supercooled water.

\section{d. Integrated lidar and radar profiles}

Figure 9a shows the scatterplot of the simulated integrated lidar profiles versus the measured ones for

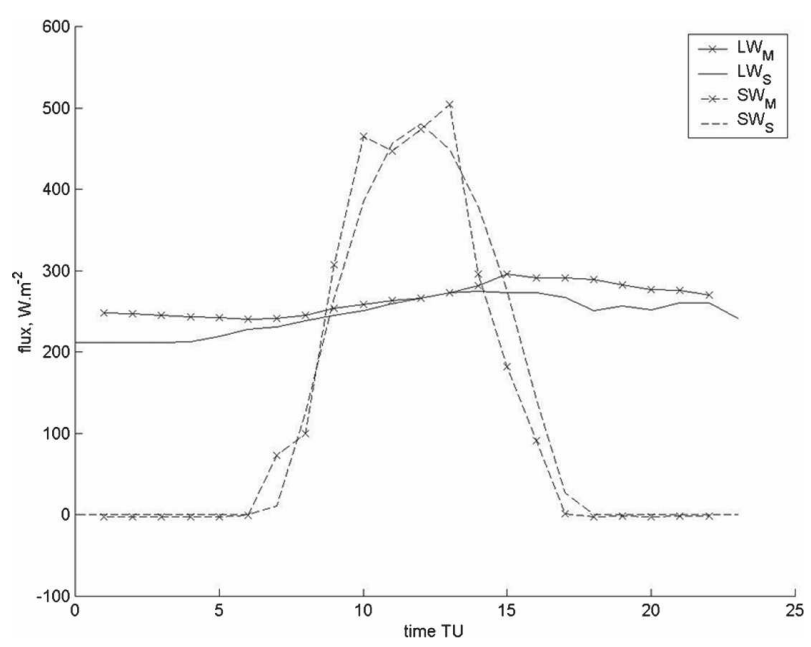

FIG. 6. Temporal evolution of fluxes for 17 Oct 2003 with the B scheme. Longwave fluxes are in solid lines and shortwave fluxes are in dashed lines. 

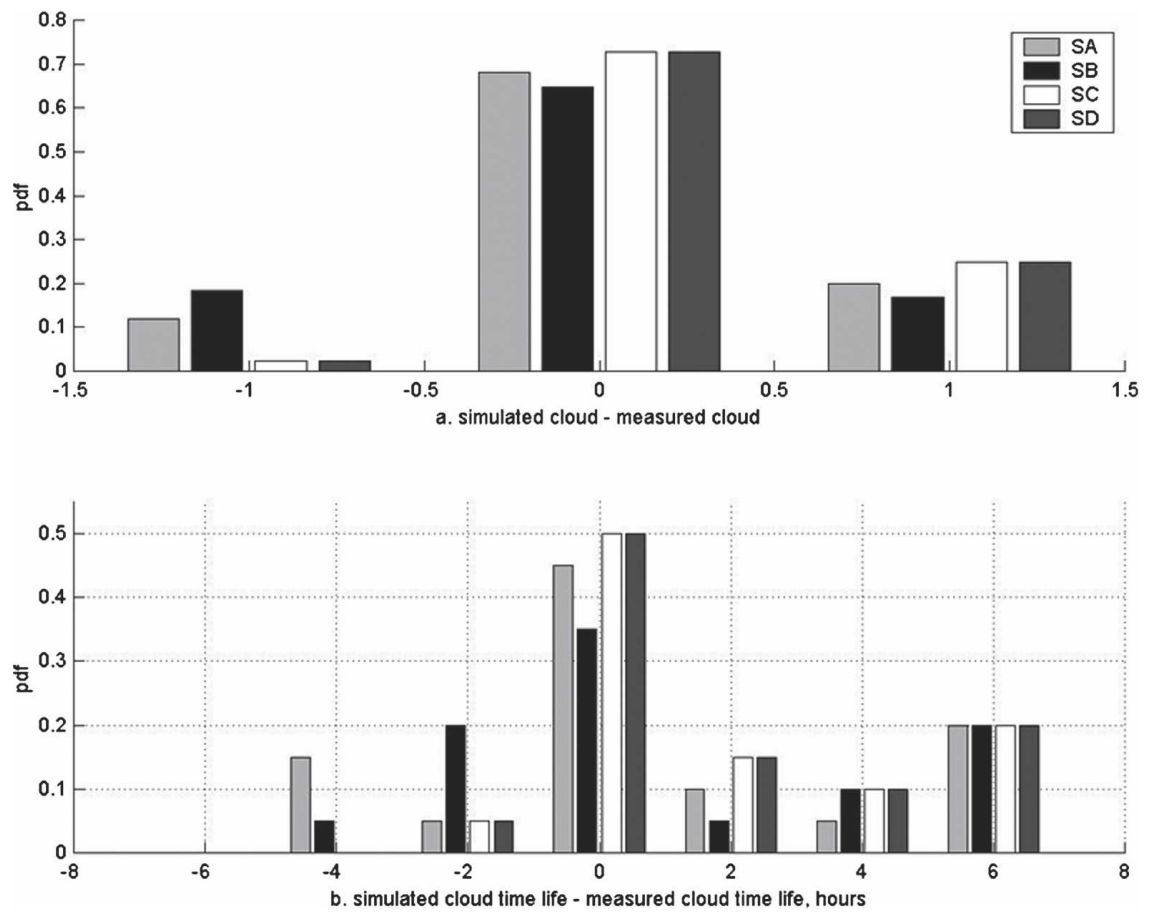

FIG. 7. Sixty-two-day statistical study of cloud occurrences and lifetimes using lidar, one point every hour for schemes (S) A-D: (a) -1 value: simulated lidar does not detect any cloud whereas observed lidar detects one; 0 value: simulated lidar and observed lidar agree concerning the presence of cloud; 1 value: simulated lidar detects a cloud whereas observed lidar does not detect any. (b) Probability density function of the difference between the simulated and the measured cloud time life, as detected by lidar (hours).

scheme $\mathrm{B}$, which is the most balanced in terms of cloud occurrence. Each integrated value is calculated from the bottom to the top of cloud. Table 3 summarizes the correlations for the different schemes. There is considerable scatter and correlations are poor, even when gathering individual points into subsets of 20 consecutive sorted simulated values and averaging over these cases. The best correlation is obtained for schemes A and $\mathrm{B}$ when considering 20-point averages $\left(R_{S / M \text {,mean }}=\right.$ $0.37-0.34$ ) but remains too low to show any skill. This large scatter is in part due to the large differences in variability of ice/snow content within the ice cloud itself, and in part to the difficulty in simulating the exact timing of the cloud, as revealed in Fig. 3 in the case of 17 October 2003. It has to be remembered that lidarcase clouds are semitransparent and generally consist of thin filament structures, which can hardly be captured with a mesoscale model.

The PDF of the difference between $\left[P_{S}(z) z^{2}\right]_{\text {integrated }}$ $\left(\mathrm{m}^{-1} \mathrm{sr}^{-1}\right)$ and $\left[P_{S}(z) z^{2}\right]_{\text {integrated }}\left(\mathrm{m}^{-1} \mathrm{sr}^{-1}\right)$ shown in Fig. $9 \mathrm{~b}$ displays a maximum near zero, proving that a significant fraction of cases are well simulated. The difference between schemes $\mathrm{A}, \mathrm{B}$, and $\mathrm{C}$ is the terminal velocity. As explained in section $2 b$ the size-dependent formulation for this fall velocity in scheme $\mathrm{C}$ may induce a positive bias in terms of ice mass because the velocity formula is applied to an average particle size. Other sources of errors can occur, such as the description of the number of particles and/or the mixing ratio in the model, or a bias in the derived particle size. It is also interesting to note that the simple ice scheme performs almost equally well as the more sophisticated A and B schemes.

Figure 9c is the same as Fig. 9a but for the integrated radar reflectivity, which is more sensitive to thicker clouds. In this case skill becomes more significant than for the lidar probably because the clouds are better defined, more massive, and therefore easier to represent at mesoscale. Table 3 shows that schemes B and D behave best. Nevertheless, Fig. 9c shows that for scheme $\mathrm{B},\left(Z d B_{M}\right)_{\text {integrated }}(d B)$ is larger than $\left(Z d B_{S}\right)_{\text {integrated }}$ $(d B)$ for small values (i.e., for thin clouds) and the reverse is true for larger values (i.e., thicker clouds). The mean bias between simulations and measurements (Fig. $9 \mathrm{~d})$ is very low compared to the other schemes and is positive, indicating that the simulated values are too large. For the three other schemes, the biases are negative, indicating that the simulated values are too small: 

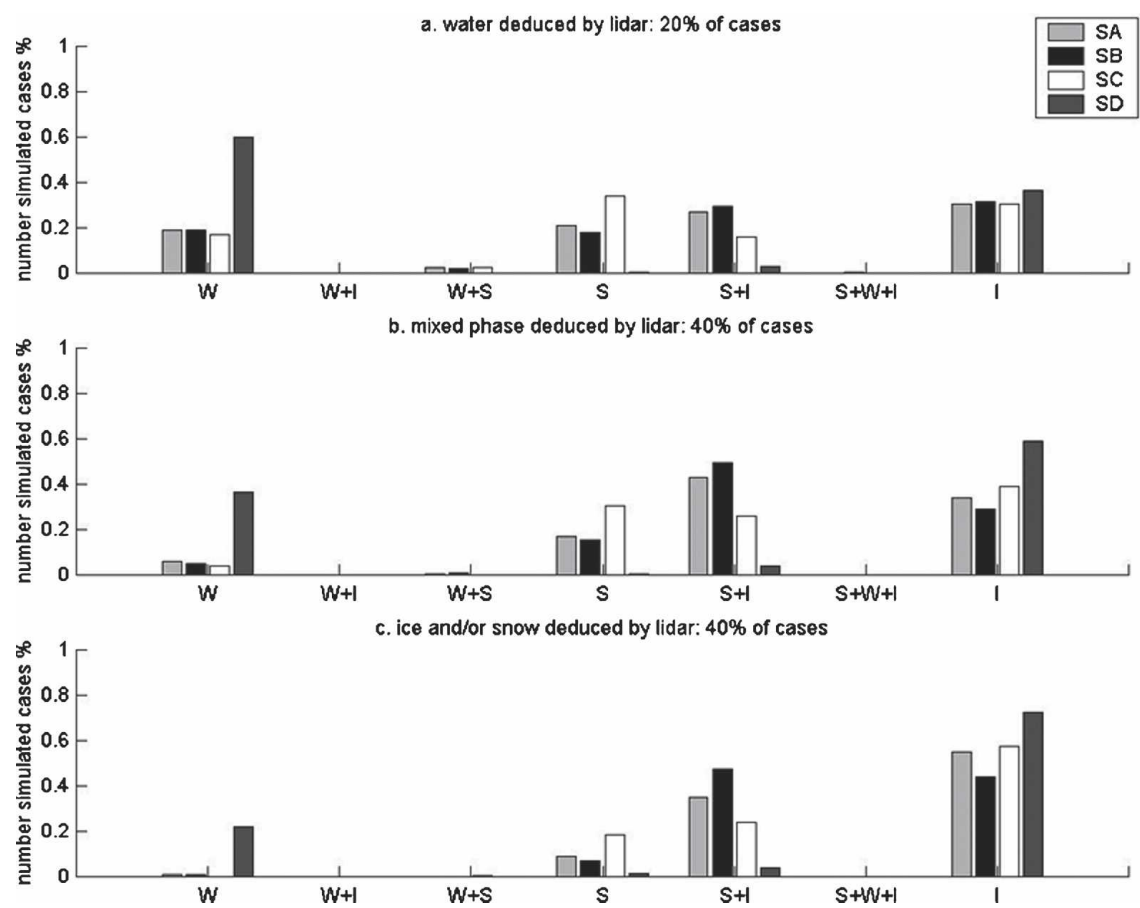

FIG. 8. Sixty-two-day statistical study about phase, one point every hour and every km for schemes (S) A-D: (a) simulated phase when measured lidar depolarization ratio leads to liquid water, (b) same as (a) but for mixed phase, and (c) same as (a) but ice. Here, W means liquid water, S means snow, and I means ice.

the number of particles (considering a constant ice/ snow mixing ratio) is too weak or the particles are too small. Schemes A and D behave in a similar manner, whereas scheme B exhibits an overestimation of radar-integrated reflectivity, but with a maxima closer to zero.

\section{e. Altitude of maximum lidar and radar signals}

We now compare the simulated and observed cloud cores, where the measured signals should be largest. Figure 10a shows the altitude of the maximum lidar signal for simulations and observations for scheme B. Although there is also a significant amount of scatter, the model appears to have skill in predicting this altitude, on average. The best nonaveraged correlation is obtained for scheme D (Table 3). Figure 10b (PDF) shows that the altitude of the simulated lidar signal maximum is about $1.2 \mathrm{~km}$ lower than the observed one for all schemes except $\mathrm{C}$.

Figure 10c shows the altitude where the radar reflectivity is maximal within the cloud, for the B scheme. Table 3 shows that the D scheme leads to the best correlation between simulations and measurements. Figure 10d shows that the simulated maximum radar signals are on average higher than the observed ones for the $\mathrm{C}$ and $\mathrm{D}$ schemes, and lower for the A and B schemes (biases in Table 3).

The model is more skilful in capturing the radar maxima variability than the lidar one (except for scheme A), as shown by the nonaveraged correlations of Table 3. The internal structure of thicker clouds seems easier to simulate than that of thinner clouds. Figure 10d shows that scheme B has nearly unbiased maxima altitudes, while scheme $\mathrm{C}$ overestimates this altitude by $2.1 \mathrm{~km}$. Since all the particles in each grid box fall at the same speed, the model produces a lack of dispersion in the vertical.

On one hand, simulated and measured altitudes of maximal radar reflectivity are in good agreement, regarding Fig. 10d and the bias values. It means that the model reproduces correctly the vertical distribution of large particle size, because the radar reflectivity depends on the sixth power of particle radius. On the other hand, the lidar backscatter signal depends on both particle size and particle concentration number at the first order. Hence, because of the result concerning radar reflectivity, the difference between simulated and measured maximal lidar signal is mostly due to a biased representation of the vertical distribution of smaller particle concentration number. Finally, a visual inspection of each case (not shown) shows that the model 

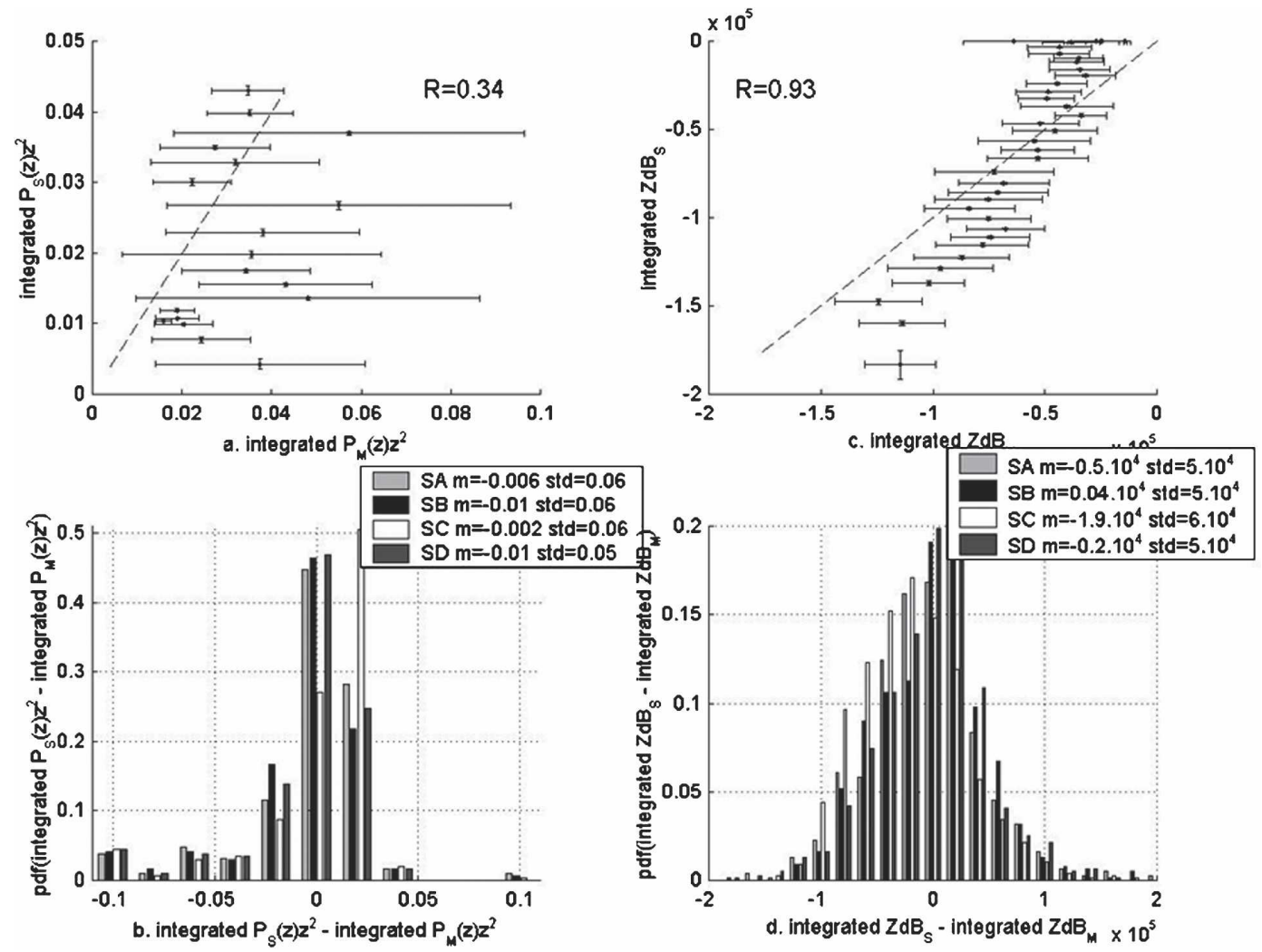

FIG. 9. Sixty-two-day statistics, one point every hour: (a) the simulated integrated lidar profiles $\left(\mathrm{m}^{-1} \mathrm{sr}^{-1}\right)$ against the measured ones, for the B scheme; each point is the mean value of the 20 closest neighbors of measured values, and error bars represent $(2 \times$ STD $) /(20)^{1 / 2}$, where STD is the standard deviation of those 20 closest neighbors; dotted line is $x=y$. (b) Probability density function of the difference between simulated and measured integrated lidar profiles for schemes (S) A-D. (c) Same as (a) but for radar reflectivity (dB). (d) Same as (b) but for radar reflectivity.

tends to overestimate the cloud height whatever the microphysical scheme.

\section{f. Radar and lidar mean profiles}

The mean profile of the complete period of observation is shown for measurements and simulations (for the four microphysical schemes) in Fig. 11a for lidar and in Fig. 11b for radar. The lidar-measured averaged profile has an important vertical variability, which is due to several cloud or aerosol layers that are very different (in terms of altitude and amplitude) from one day to another. The peak at $2 \mathrm{~km}$ is due to an aerosol layer that is present almost every day over the SIRTA ground-based site. This peak does not influence the computation of the height of the maximum lidar signal for two reasons: (i) when profiles are not averaged, this 2-km signal is inferior to the signal that is backscattered by a cloud; (ii) the detection of the height of the maximum lidar signal is done only within the cloud, and the boundaries of the cloud are determined by the STRAT algorithm, which distinguishes aerosol and cloud layers. Finally, the measured lidar signal is not inversed and is normalized using a simple method: by forcing the signal to be equal to a molecular signal in a cloud-free area. Hence, the differences observed between the simulated and the measured lidar averaged profiles (Fig. 11a) are only due to the model.

The shape of the curves confirms the precedent results and is in good agreement with Figs. 9 and 10: for the four schemes, it is difficult to reproduce the lidar profile. Nevertheless, the profile from scheme $\mathrm{C}$ is by the same order of magnitude than the measured one, but with a higher and more extended cloud. The simulated mean radar profiles using schemes A and B are close to the measured one, in terms of order of magnitude and in terms of altitude, meaning that the vertical distribution of the particle size is good, and so the lidar discrepancies are mostly due to a problem of the vertical distribution of the particle concentration number. 
TABLE 3. Correlation coefficients between simulated and measured values, for all considered variables: $R_{S / M}$ are coefficients for global values and $R_{S / M \text {,mean }}$ (italic) for values averaged every 20 closest points. The indicated bias (bold) is the mean value of the difference between the simulated and the measured variable.

\begin{tabular}{|c|c|c|c|c|c|c|c|c|}
\hline & $\begin{array}{c}R_{S / M} \\
R_{S / M \text {,mean }} \\
\quad \text { for A }\end{array}$ & $\begin{array}{l}\text { Bias } \\
\text { for A }\end{array}$ & $\begin{array}{c}R_{S / M} \\
R_{S / M \text {,mean }} \\
\quad \text { for B }\end{array}$ & $\begin{array}{l}\text { Bias } \\
\text { for B }\end{array}$ & $\begin{array}{c}R_{S / M} \\
R_{S / M \text {,mean }} \\
\quad \text { for C }\end{array}$ & $\begin{array}{l}\text { Bias } \\
\text { for C }\end{array}$ & $\begin{array}{c}R_{S / M} \\
R_{S / M \text {,mean }} \\
\quad \text { for D }\end{array}$ & $\begin{array}{l}\text { Bias } \\
\text { for D }\end{array}$ \\
\hline $\begin{array}{c}{\left[P(z) z^{2}\right]_{\text {integrated }}} \\
\left(\mathrm{m}^{-1} \mathrm{sr}^{-1}\right)\end{array}$ & $\begin{array}{l}0.12 \\
0.37\end{array}$ & -0.006 & $\begin{array}{l}0.16 \\
0.34\end{array}$ & -0.01 & $\begin{array}{l}0.03 \\
0.14\end{array}$ & -0.002 & $\begin{array}{l}0.08 \\
0.16\end{array}$ & -0.01 \\
\hline $\begin{array}{l}z\left[P(z) z^{2}\right]_{\max } \\
\quad(\mathrm{m})\end{array}$ & $\begin{array}{l}0.13 \\
0.22\end{array}$ & -0.66 & $\begin{array}{l}0.12 \\
0.26\end{array}$ & -1.2 & $\begin{array}{l}0.13 \\
0.30\end{array}$ & 0.51 & $\begin{array}{l}0.22 \\
0.46\end{array}$ & -1.3 \\
\hline $\begin{array}{l}(\mathrm{dB} Z)_{\text {integrated }} \\
\quad(\mathrm{dB})\end{array}$ & $\begin{array}{l}0.50 \\
0.87\end{array}$ & $-0.5 \times 10^{4}$ & $\begin{array}{l}0.51 \\
0.93\end{array}$ & $0.04 \times 10^{4}$ & $\begin{array}{l}0.40 \\
0.86\end{array}$ & $-1.9 \times 10^{4}$ & $\begin{array}{l}0.57 \\
0.93\end{array}$ & $-0.22 \times 10^{4}$ \\
\hline $\begin{array}{l}Z(\mathrm{~dB} Z)_{\max } \\
(\mathrm{m})\end{array}$ & $\begin{array}{l}0.051 \\
0.63\end{array}$ & -0.13 & $\begin{array}{l}0.17 \\
0.35\end{array}$ & -0.02 & $\begin{array}{l}0.16 \\
0.20\end{array}$ & 2.1 & $\begin{array}{l}0.39 \\
0.80\end{array}$ & 0.44 \\
\hline $\begin{array}{c}V(\mathrm{dBZ})_{\max } \\
\left(\mathrm{m} \mathrm{s}^{-1}\right)\end{array}$ & $\begin{array}{l}0.08 \\
0.68\end{array}$ & -0.10 & $\begin{array}{l}0.08 \\
0.25\end{array}$ & 0.50 & $\begin{array}{l}0.13 \\
0.13\end{array}$ & -0.19 & $\begin{array}{l}0.07 \\
0.72\end{array}$ & 0.40 \\
\hline $\begin{array}{l}\mathrm{LW} \\
\qquad\left(\mathrm{W} \mathrm{m}^{-2}\right)\end{array}$ & $\begin{array}{c}0.77 \\
-\end{array}$ & -10 & $\begin{array}{c}0.76 \\
-\end{array}$ & -13 & $\begin{array}{c}0.76 \\
-\end{array}$ & -8 & $\begin{array}{c}0.79 \\
-\end{array}$ & -12 \\
\hline $\begin{array}{l}\text { SW } \\
\qquad\left(\mathrm{W} \mathrm{m}^{-2}\right)\end{array}$ & $\begin{array}{r}0.89 \\
-\end{array}$ & 12 & $\begin{array}{c}0.89 \\
-\end{array}$ & 28 & $\begin{array}{c}0.87 \\
-\end{array}$ & -45 & $\begin{array}{c}0.9 \\
-\end{array}$ & 17 \\
\hline
\end{tabular}

Furthermore, it is interesting to notice that the simple scheme D performs as well as schemes A and B in the highest parts of the clouds.

\section{g. Doppler velocity}

Figure 12a shows the Doppler velocity at the altitude where radar reflectivity is maximum $V\left(\mathrm{~dB} Z_{M / S}\right)_{\max }$ $\left(\mathrm{m} \mathrm{s}^{-1}\right)$, for scheme B. We choose to look at the altitude of maximal radar reflectivity because it must correspond to the location of the largest particles. The simulated Doppler velocities have absolute values smaller than the measured ones. Actually, the formulation of the monocrystal terminal fall velocity $u_{t, \text { ice }}$ that comes from Heymsfield and Donner (1990) and Heymsfield (1972) becomes unphysical for $r_{\text {e.ice }}>450$ $\mu \mathrm{m}$ because it decreases, whereas it should still increase. Furthermore, $r_{\text {e.ice }}>450 \mu \mathrm{m}$ for a significant part of the dataset (almost 20\%; not shown). For those $20 \%$ of cases, the terminal fall velocity will be unphysically small. This result explains the fact that for schemes $\mathrm{B}$ and $\mathrm{D}$, the simulated Doppler velocity is very small compared to the measured one. Scheme A produces the best correlations (Table 3). As shown by Fig. 12b and Table 3, schemes B, C, and D underestimate the absolute value of the velocity, and it is the contrary for scheme A.

Because of the weak order of magnitude of $w_{S}$ compared to $V_{S, t}$, general biases are mostly due to the difference between the simulated and the measured terminal velocities: simulated particles fall more slowly than observed ones for schemes B, C, and D. This is consistent with the earlier conclusion that the cloud lifetime was longer for simulations than for observations. However one should keep in mind that only the large particles in the particle size distribution can be seen by the radar and therefore the fall velocity of smaller, but more numerous particles is not accounted for. The discrepancies between simulations and observations are consistent with this possible effect.

Even though scheme $\mathrm{C}$ is the most consistent with the assumptions in the Reisner et al. (1998) microphysical scheme (terminal fall velocity related to particle size), it reveals the largest bias with simulated velocities underestimated by $0.6 \mathrm{~cm} \mathrm{~s}^{-1}$. For the A scheme, the overestimation of the Doppler velocity is not consistent with the too long lifetime, meaning that the Doppler velocity is not well calculated for those two schemes in ACTSIM.

Scheme B, which relates the terminal fall velocity to ice mixing ratio, shows less bias. This is consistent with results found for the altitude of maximal radar reflectivity that was also less biased for this scheme.

\section{h. Radiative fluxes}

Figures 13a and $13 \mathrm{c}$ show the simulated fluxes as a function of the measured ones, for scheme B. The skill in predicting cloud occurrence is reflected by the skill in predicting radiative fluxes. All schemes give similar correlation coefficients (Table 3). However differences can be seen: Fig. 13b shows that the longwave flux is on average underestimated for all schemes (of about $10 \mathrm{~W}$ $\mathrm{m}^{-2}$, as shown in Table 3), but in particular for the smallest values, which could correspond to the clearsky situation. This is consistent with results for the il- 

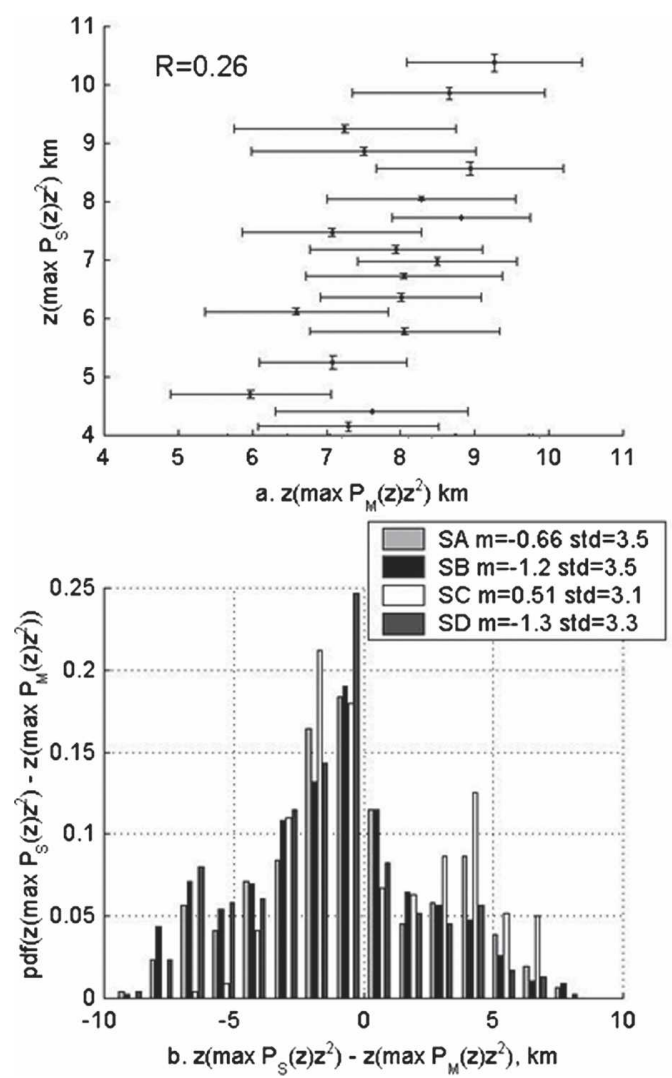
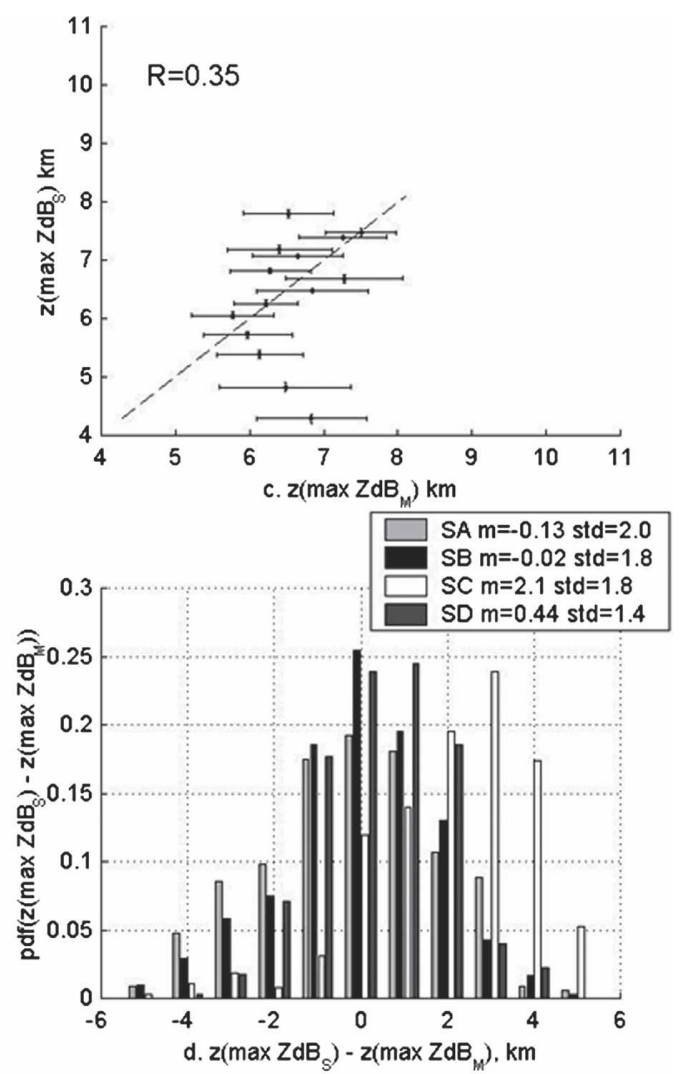

FIG. 10. Sixty-two-day statistics, one point every hour: (a) same as Fig. 9a but for the altitude where lidar signal is maximum, (b) same as Fig. 9b but for the altitude where lidar signal is maximum, (c) same as (a) but for the altitude where radar reflectivity is maximum, and (d) same as (b) but for the altitude where the radar reflectivity is maximum.

lustrative example (section 5): the clear-sky LW fluxes are not accurate. This could either be due to errors in simulated cloud properties or to clear-sky simulated LWF bias, because the latter is known to be difficult to accurately simulate in atmospheric models. On the contrary, the simulated SWF is overestimated for all schemes except scheme C (bias values in Table 3). As the radiative code is the same for all model experiments, the discrepancy is due the microphysical scheme. For schemes A, B, and D, the longwave and shortwave fluxes behaviors are self-consistent and are in agreement with the lidar and radar integrated signals to show that the simulated cloud is not opaque enough.

\section{Discussion and conclusions}

\section{a. Limits of the method}

\section{1) IMPACT OF MICROPHYSICAL SCHEMES HYPOTHESIS}

The four microphysical schemes assume the ice/snow particles shape to be spherical and constant within the cloud (no vertical variability), which is unrealistic, in particular for thin clouds that are only detected by lidar. These hypotheses may significantly influence the lidar simulated profile and can explain a significant part of the differences between the simulated and the measured lidar maxima and integrated profiles, considering a vertical variability of the particle shape would change the values of $\beta_{\text {ice }}$ and $\alpha_{\text {ice }}$ that influence the vertical variability of the lidar signal [Eq. (3)], and consequently the altitude where the lidar signal is maximal. Furthermore, this could also influence the radiative effect of the clouds; for example, a cloud composed of plates instead of ice spheres will increase the albedo by $19.5 \%$ (Liou 1986).

\section{2) IMPACT OF ACTSIM HYPOTHESIS}

Table 2 shows that for scheme $\mathrm{D}, r_{S \text {,ice }}$ must be fixed in ACTSIM and $N_{S \text {,ice }}$ is calculated as a function of $1 / r_{S, \text { ice }}^{3}$ : the radar reflectivity is then proportional to $r_{S, \text { ice }}^{3}$ as shown by (11), whereas lidar signal is proportional to $1 / r_{S, \text { ice }}^{2}$ as shown by (3) and (7). Hence, for this scheme, 

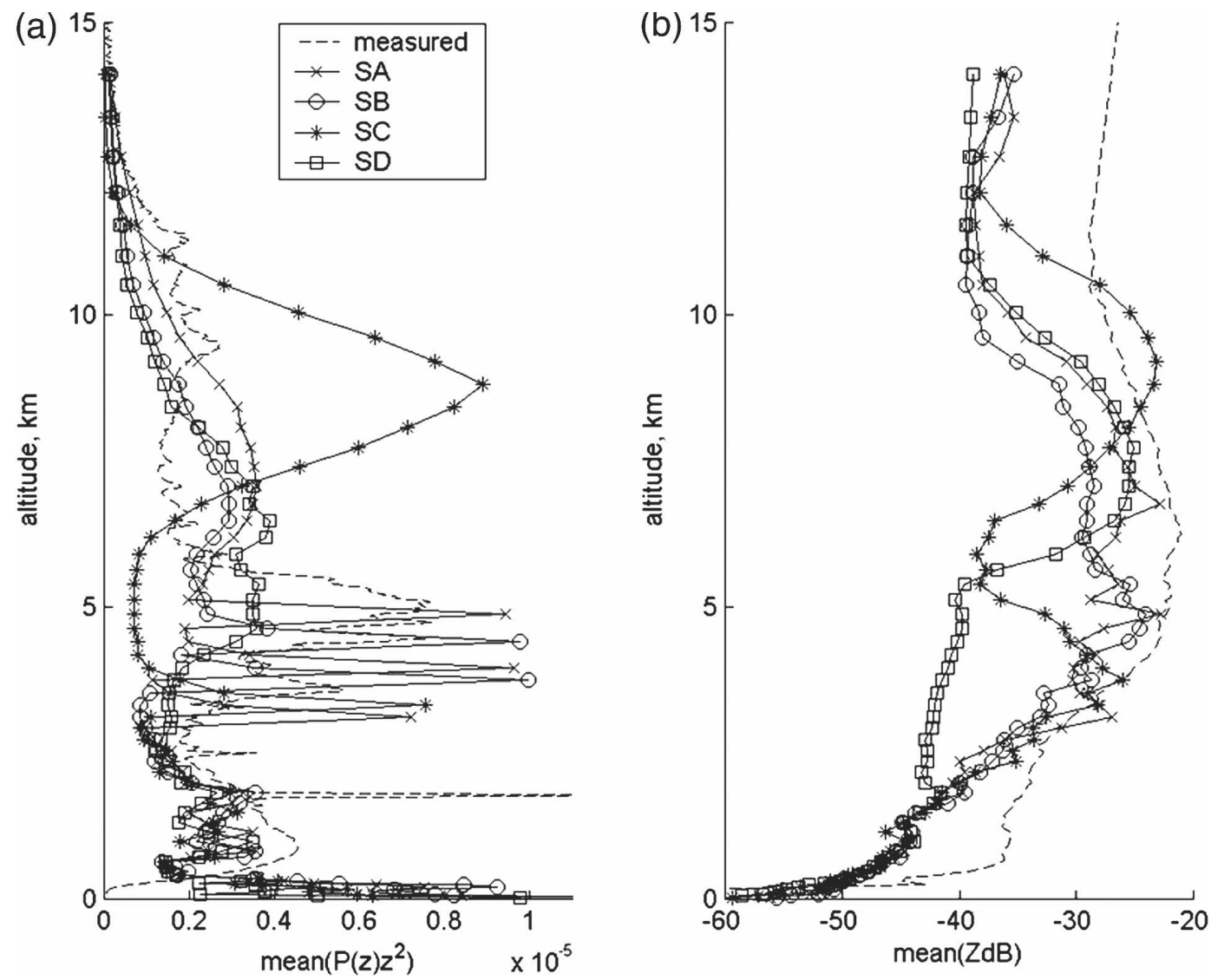

FIG. 11. (a) Mean profile of all lidar profiles $\left(\mathrm{m}^{-1} \mathrm{sr}^{-1}\right)$ (every selected case every hour): measurements (dashed line), scheme A simulation (line with crosses), scheme B (line with circles), scheme C (line with stars), and scheme D (line with squares). (b) Same as (a) but for radar reflectivity (dB).

lidar and radar signals vary inversely when $r_{\mathrm{S} \text {,ice }}$ varies: if the fixed value of $r_{\mathrm{S} \text {, ice }}$ is too large, the radar reflectivity will also be too large, whereas the lidar signal will be too weak.

One can notice that only terminal fall velocity of ice crystals (and not that of snow particles) is considered in the formulation of the Doppler velocity (12): actually this variable is the only difference between schemes $\mathrm{A}$, $\mathrm{B}$, and $\mathrm{C}$. Hence, one can understand how this difference is responsible for the differences of mass content, occurrence, lifetime, and fluxes.

\section{3) Cloud spatial variability}

Figure 1 illustrates the possible spatial heterogeneities around SIRTA concerning the total column of water content. This spatial variability explains the difficulty to perfectly hit the cloud time sequence, and therefore probably accounts for a significant part of the difference between simulations and measurements concerning the integrated lidar signal, but statistically these differences should average out to zero. The quasisystematic overestimation of the cloud lifetime in simulations for all microphysical schemes is not due to this problem.

\section{4) Sensitivity to THE MODEL FORCING}

The MM5 model is forced by lateral boundary conditions in wind, temperature, but also water vapor mixing ratio. These boundary conditions obviously influence the quality of the simulation. It is not clear however what part of the simulations skill is due to the "internal" microphysics, or to the transport of humidity from lateral boundaries. Since there are not liquid/solid water lateral inputs, these phases are entirely created by the internal microphysics. Moreover our results show significant differences between the results obtained from the four schemes tested above. All these arguments indicate that most of the skill should be attributable to the subgrid processes, in particular the internal microphysics. 

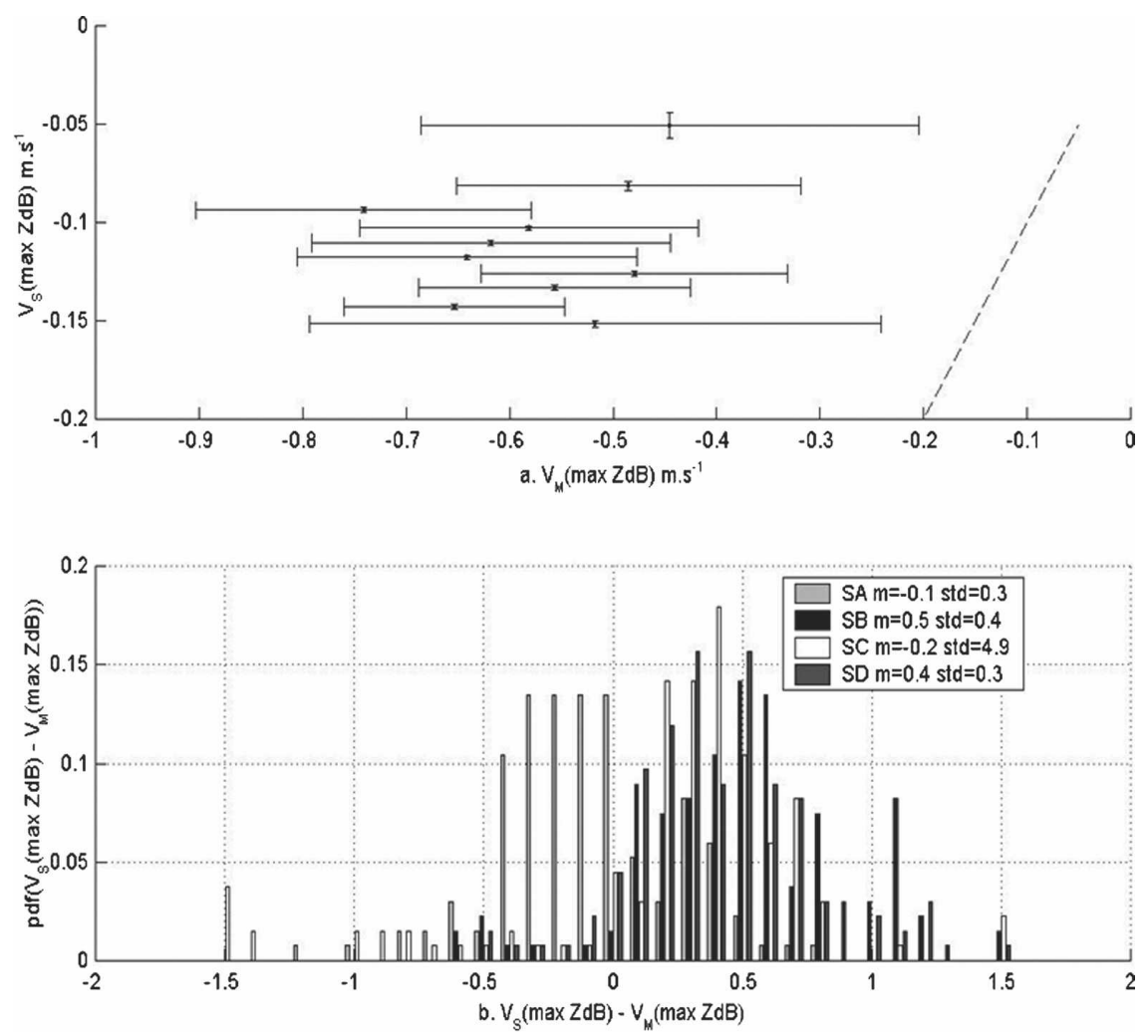

FIG. 12. Sixty-two-day statistical study of vertical velocity, one point every hour: (a) simulated velocity against measured one, at the altitude of maximum radar reflectivity, for the $\mathrm{B}$ scheme; downward velocities are negative numbers. (b) Probability density function of the difference between simulated and measured velocity at the altitude of maximum radar reflectivity $\left(\mathrm{m} \mathrm{s}^{-1}\right)$ for schemes (S) A-D. A positive bias suggests that the simulated vertical velocities are underestimated.

\section{b. Microphysical scheme evaluation}

The general tendencies are drawn in Table 4. In terms of systematic biases, all microphysics tend to produce clouds that are too persistent (occurrence and lifetime). Scheme B, which consists of Reisner et al. (1998) microphysics with the Heymsfield and Donner (1990) terminal velocity expression is the least biased scheme. The original Reisner et al. (1998) scheme (C) has the strongest bias. The model tends to produce ice and snow instead of liquid water (thermodynamical phase), and when ice clouds are properly built, they tend to contain an insufficient quantity of ice/snow (integrated lidar and radar signals and SWF), that is, to not be opaque enough. The cloud height is overestimated in the model (altitude of lidar and radar maxima). All those conclusions about the cloud macrophysical properties that are almost similar for the four microphysical schemes could be a signature of the difficulty in reproducing the subgrid-scale thermodynamical processes such as turbulence and convection.
The strong bias of the Reisner et al. (1998) scheme makes it difficult to use in practice. The small modifications of the velocities clearly improve the model results in terms of cloud occurrence and radiation. The "simple ice" scheme has striking skill given its degree of simplification, and has the advantage of being computer run time inexpensive but does not provide water phase characteristics. The modifications of Reisner et al. (1998) proposed in schemes A and B correct most of the biases of scheme C. Other terminal velocity parameterizations could be tested in the future (i.e., Heymsfield et al. 2002) when the particle nonsphericity will be taken into account in the model, as it is possible to compute it in ACTSIM code. Finally, the results about the performance of the simple ice scheme (Dudhia 1989) show that this is not only the microphysical scheme that influences the ability to simulate ice clouds, but also other subgrid-scale processes such as the convection scheme.

Table 5 shows the correlation between simulations and measurements for the different cloud classes (de- 

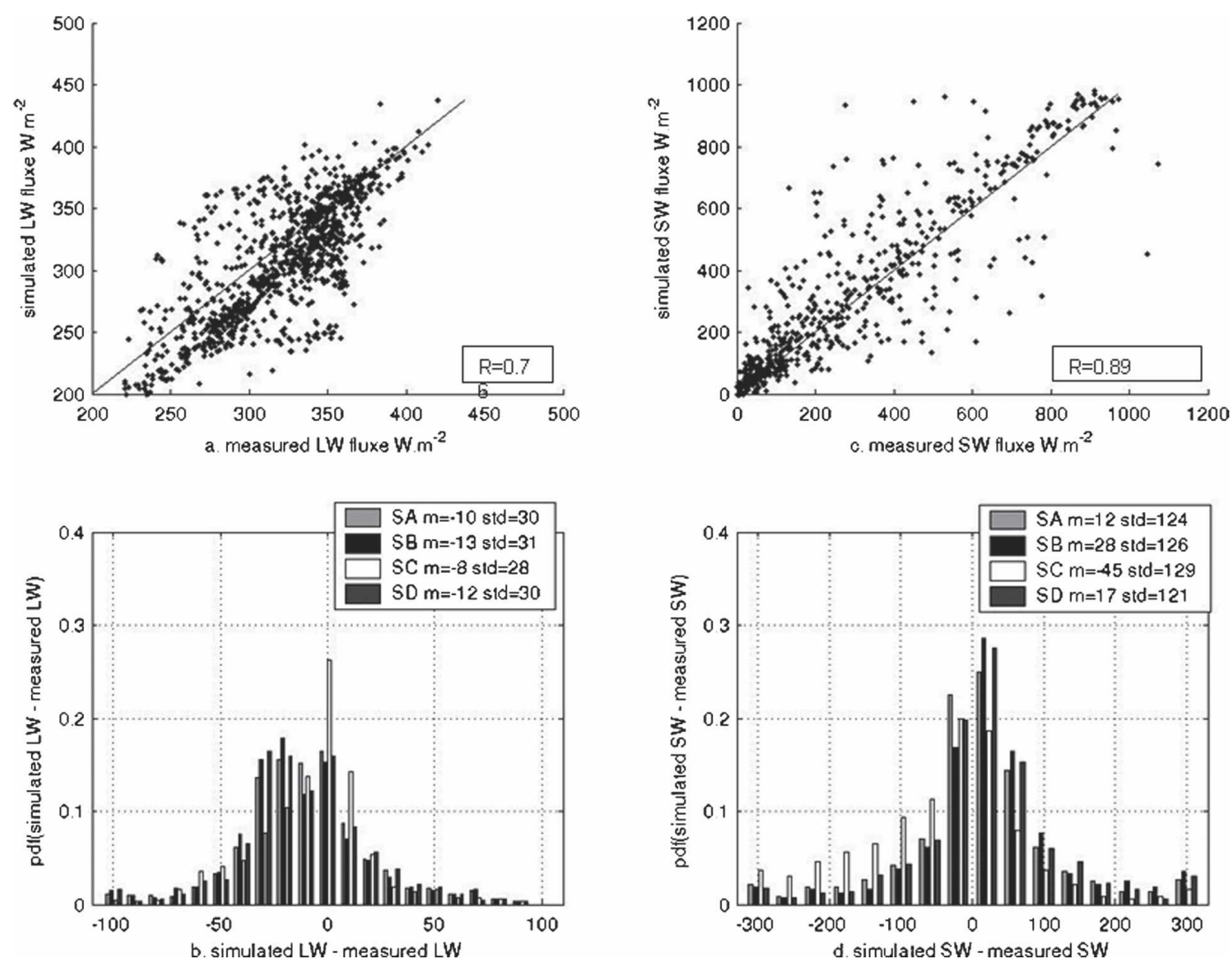

FIG. 13. Sixty-two-day statistical study of fluxes, one point every hour: (a) simulated longwave flux against measured one for the B scheme; (b) probability density function of the difference between the simulated and the measured longwave flux $\left(\mathrm{W} \mathrm{m}^{-2}\right.$ ) for schemes (S) A-D; (c) same as (a) but for shortwave flux; and (d) same as (b) but for shortwave flux $\left(\mathrm{W} \mathrm{m}^{-2}\right)$.

fined in section $3 \mathrm{~b}$ ). Scheme B is less accurate for thin clouds than for medium and thick clouds, according to integrated lidar and radar profiles, and SWF. This could be due to the following: (i) the complex vertical structure of thin clouds that are difficult to reproduce in the model grid; (ii) the hypothesis of spherical particles that could be more justified for thick clouds as particles are often larger in this type of clouds, and as was noted in section $7 \mathrm{a}(1)$, it could have a strong influence on the lidar signal and the fluxes; (iii) the thermodynamical

TABLE 4. Best microphysical scheme performance for each variable concerning the correlation coefficient, the mean value of the difference between simulations and measurements, and the tendency; "simu" stands for simulations, and "measu" for measurements.

\begin{tabular}{|c|c|c|c|}
\hline & $\begin{array}{l}\quad R_{S / M} \\
\text { best scheme }\end{array}$ & $\begin{array}{l}\text { Mean (simu-measu) } \\
\text { best scheme }\end{array}$ & General tendency \\
\hline Occurrence & - & $\mathrm{B}$ & Too much occurrence for A, C, and D; B more equilibrate \\
\hline Lifetime & - & All equivalent & Clouds too persistent \\
\hline Thermodynamical phase & - & All equivalent & Too much ice and snow and not enough water \\
\hline$\left[P(z) z^{2}\right]_{\text {integrated }}$ & $\mathrm{B}$ & $\mathrm{A}, \mathrm{C}$ & Simu $<$ measu, but "bad scores" for all schemes \\
\hline$(\mathrm{dB} Z)_{\text {integrated }}$ & $\mathrm{B}, \mathrm{D}$ & $\mathrm{B}$ & Simu $<$ measu except for $\mathrm{B}$ \\
\hline$z\left[P(z) z^{2}\right]_{\max }$ & $\mathrm{D}$ & $\mathrm{C}$ & Simu $<$ measu except for $\mathrm{C}$ \\
\hline$z(\mathrm{dBZ})_{\max }$ & $\mathrm{D}$ & $\mathrm{B}$ & Simu $>$ measu for $\mathrm{C}, \mathrm{DSimu}<$ measu for $\mathrm{A}, \mathrm{B}$ \\
\hline$V(\mathrm{~dB} Z)_{\max }$ & A & A & $\begin{array}{l}\mid \text { simu }|>| \text { measu } \mid \text { for A, C|simu }|>| \text { measu } \mid \text { for B, D, but } \\
\text { "bad scores" for all schemes }\end{array}$ \\
\hline LW & All equivalent & All equivalent & Simu $<$ measu \\
\hline SW & All equivalent & A & Simu $>$ measu except for $\mathrm{C}$ \\
\hline
\end{tabular}


TABLE 5. Correlation coefficients between simulated and measured values as a function of the cloud class, for the B scheme.

\begin{tabular}{lcccc}
\hline \hline & $\begin{array}{c}R_{S / M} \\
\text { thin } \\
\text { cloud }\end{array}$ & $\begin{array}{c}R_{S / M} \\
\text { medium } \\
\text { cloud }\end{array}$ & $\begin{array}{c}R_{S / M} \\
\text { thick } \\
\text { cloud }\end{array}$ & $\begin{array}{c}R_{S / M} \\
\text { medium } \\
+ \text { thick } \\
\text { (radar only) }\end{array}$ \\
\hline$\left[P(z) z^{2}\right]_{\text {integrated }}$ & 0.039 & 0.18 & - & - \\
$(\mathrm{dB} Z)_{\text {integrated }}$ & - & 0.42 & 0.59 & 0.22 \\
LW & 0.83 & 0.68 & 0.61 & 0.47 \\
SW & 0.91 & 0.92 & 0.91 & 0.97 \\
\hline
\end{tabular}

structure that creates the conditions for the existence of clouds is different for thin clouds and medium/thick clouds and could be better represented for medium clouds than thin clouds in the model.

In other statistical calculations (not shown) we have attempted to study the seasonal dependence of the model skill. Although this requires probably more than $1 \frac{1}{2}$ years of data to fully confirm, we did not find seasonal variation.

\section{c. General conclusions}

The relatively large number of cloud cases studied here leads to a possible statistical study of the ability of MM5 to simulate the active remote sensing variables. The first important result of this study is the ability of the MM5 model to simulate ice clouds. The four schemes simulate too persistent clouds as compared to observations. The thermodynamical phase is either not considered (scheme D) or poorly reproduced. The results are very sensitive to the formulation of the terminal velocity. The scheme based on Reisner et al. (1998) with Heymsfield and Donner (1990) terminal velocity is the most skilful, on average, in simulating midlatitude ice clouds with medium optical depth.

Additionally, information about the true microphysical properties (particle size and shape, density) would certainly help assess in which microphysical conditions the MM5 model associated with scheme B are most accurate. With this conclusion in mind, a microphysical study will be performed using the same type of measurements and adding ice cloud microphysical retrievals: ice particle shape retrieval using Noel et al. (2002), ice particle size from lidar and IR radiometry (Chiriaco et al. 2004), from radar-lidar combination (Tinel et al. 2005), and from radar only (Protat et al. 2004), as well as liquid and ice water content and fluxes at the top of the atmosphere applied to the geostationary Meteosat Second Generation (MSG) satellite (Minnis et al. 1998). The approach presented in this paper will also be carried on using Aqua Train spaceborne observations
[Cloud-Aerosol Lidar and Infrared Pathfinder Satellite Observation (CALIPSO) and CloudSat] to evaluate cloud model performance in selected areas.

Acknowledgments. Thanks are due to C. Mass for the fruitful discussion and his suggestion concerning the use of the radiative scheme. Thanks are also due to the SIRTA scientific team and technical staff for providing and preparing the dataset, to people involved in the development of MM5, and to the COMPERES project for running and preparing models outputs.

\section{APPENDIX}

\section{List of Symbols}

\begin{tabular}{|c|c|c|}
\hline Notation & Description & Unit \\
\hline$D_{\mathrm{eq}}$ & Equivalent diameter & $\mathrm{m}$ \\
\hline$K_{\text {snow/ice/liquid }}^{2}$ & $\begin{array}{l}\text { Dielectric factor of snow/ice/ } \\
\text { liquid }(=0.176 \text { for ice and } \\
\text { snow and }=0.930 \text { for liquid } \\
\text { water, at } 94 \mathrm{GHz})\end{array}$ & - \\
\hline $\mathrm{LW}_{n}$ & Net longwave flux & $\mathrm{W} \mathrm{m}^{-2}$ \\
\hline$N_{\text {ice/snow/liquid }}$ & $\begin{array}{l}\text { Ice/snow/liquid particle number } \\
\text { concentration }\end{array}$ & $\mathrm{m}^{-3}$ \\
\hline$N_{0, \text { snow }}$ & $\begin{array}{l}\text { Intercept slope parameter of the } \\
\text { Marshall-Palmer distribution }\end{array}$ & $\mathrm{m}^{-4}$ \\
\hline$n_{\text {ice/snow/liquid }}(\Re) d \Re$ & $\begin{array}{l}\text { Number of particles of ice/snow/ } \\
\text { liquid that have a radius } \\
\text { between } \Re \text { and } \Re+d \Re(\mathrm{m})\end{array}$ & $\mathrm{m}^{-4}$ \\
\hline$p$ & Pressure of air & $\mathrm{hPa}$ \\
\hline$P_{\pi, \text { snow/ice/liquid }}$ & $\begin{array}{l}\text { Scattering phase function in } \\
\text { backscattering by snow/ice/liquid }\end{array}$ & - \\
\hline$P(z) z^{2}$ & $\begin{array}{l}\text { Lidar backscattered normalized } \\
\text { signal at the altitude } \mathrm{z}\end{array}$ & $\mathrm{m}^{-1} \mathrm{sr}^{-1}$ \\
\hline$\left[P_{S / M}(z) z^{2}\right]_{\text {integrated }}$ & $\begin{array}{l}\text { Simulated/measured integrated } \\
\text { lidar signal }\end{array}$ & $\mathrm{m}^{-1} \mathrm{sr}^{-1}$ \\
\hline$Q$ & $\begin{array}{l}\text { Particle scattering efficiency } \\
(=2)\end{array}$ & - \\
\hline$q_{\text {ice/snow/liquid }}$ & Ice/snow/liquid mixing ratio & $\mathrm{kg} \mathrm{kg}^{-1}$ \\
\hline$r_{\text {liquid/snow/ice }}$ & $\begin{array}{l}\text { Radius of a particle of mean } \\
\text { mass of liquid/snow/ice }\end{array}$ & $\mathrm{m}$ \\
\hline$R_{S / M \text {,mean }}$ & $\begin{array}{l}\text { Correlation coefficient between } \\
\text { simulations and measurements, } \\
\text { using averaged values }\end{array}$ & - \\
\hline$R_{S / M}$ & $\begin{array}{l}\text { Correlation coefficient between } \\
\text { simulations and measurements }\end{array}$ & \\
\hline $\mathrm{SW}_{\mathrm{dct}}$ & Direct shortwave flux & $\mathrm{W} \mathrm{m}^{-2}$ \\
\hline $\mathrm{SW}_{\mathrm{dff}}$ & Diffuse shortwave flux & $\mathrm{W} \mathrm{m}^{-2}$ \\
\hline SW & Total downward shortwave flux & $\mathrm{W} \mathrm{m}^{-2}$ \\
\hline $\mathrm{T}$ & Temperature of air & $\mathrm{K}$ \\
\hline$T_{t}$ & $\begin{array}{l}\text { Threshold temperature of } \\
\text { conversion between liquid and } \\
\text { solid water }\end{array}$ & ${ }^{\circ} \mathrm{C}$ \\
\hline$u_{t}$ & $\begin{array}{l}\text { Mono-crystal terminal fall } \\
\text { velocity }\end{array}$ & $\mathrm{m} \mathrm{s}^{-1}$ \\
\hline$V$ & Doppler velocity & $\mathrm{m} \mathrm{s}^{-1}$ \\
\hline$V_{t}$ & $\begin{array}{l}\text { Terminal fall velocity of cloud } \\
\text { particles }\end{array}$ & $\mathrm{m} \mathrm{s}^{-1}$ \\
\hline
\end{tabular}




\begin{tabular}{|c|c|c|}
\hline Notation & Description & Unit \\
\hline$V\left(\mathrm{~dB} Z_{S / M}\right)_{\max }$ & $\begin{array}{l}\text { Simulated/measured Doppler } \\
\text { velocity where the radar } \\
\text { reflectivity is maximal }\end{array}$ & $\mathrm{m} \mathrm{s}^{-1}$ \\
\hline$v_{t}$ & $\begin{array}{l}\text { Reflectivity weighted terminal } \\
\text { velocity of the ice particles }\end{array}$ & $\mathrm{m} \mathrm{s}^{-1}$ \\
\hline$w$ & Vertical velocity of air & $\mathrm{m} \mathrm{s}^{-1}$ \\
\hline$z$ & Altitude & $\mathrm{km}$ \\
\hline $\mathrm{dB} Z$ & Radar reflectivity & $\mathrm{dB}$ \\
\hline$Z_{\text {snow/ice/liquid }}$ & $\begin{array}{l}\text { Contribution of snow/ice/liquid } \\
\text { to the radar reflectivity }\end{array}$ & $\mathrm{m}^{3}$ \\
\hline$\left(\mathrm{dB} Z_{S / M}\right)_{\text {integrated }}$ & $\begin{array}{l}\text { Simulated/measured integrated } \\
\text { radar reflectivity }\end{array}$ & $\mathrm{dB}$ \\
\hline$\alpha_{\mathrm{par} / \text { snow/ice/liquid }}$ & $\begin{array}{l}\text { Particle/snow/ice/liquid } \\
\text { attenuation by scattering }\end{array}$ & $\mathrm{m}^{-1}$ \\
\hline$\beta_{\mathrm{par} / \text { snow/ice/liquid }}$ & $\begin{array}{l}\text { Lidar backscattering coefficient } \\
\text { by particles/snow/ice/liquid }\end{array}$ & $\mathrm{m}^{-1} \mathrm{sr}^{-1}$ \\
\hline$\beta_{\mathrm{mol}}$ & $\begin{array}{l}\text { Lidar backscattering coefficient } \\
\text { by molecules }\end{array}$ & $\mathrm{m}^{-1} \mathrm{sr}^{-1}$ \\
\hline$\eta$ & $\begin{array}{l}\text { Multiple scattering correction } \\
\text { parameter }(=0.5)\end{array}$ & - \\
\hline$\rho_{\text {air }}$ & Density of air & $\mathrm{kg} \mathrm{m}^{-3}$ \\
\hline$\rho_{\text {liq/ice/snow }}$ & $\begin{array}{l}\text { Density of liquid water/ice/snow } \\
\left(=1000 \mathrm{~kg} \mathrm{~m}^{-3} / 500 \mathrm{~kg} \mathrm{~m}^{-3} / 100\right. \\
\left.\mathrm{kg} \mathrm{m}^{-3}\right)\end{array}$ & $\mathrm{kg} \mathrm{m}^{-3}$ \\
\hline$\theta$ & Solar zenith angle & $\mathrm{rad}$ \\
\hline
\end{tabular}

\section{REFERENCES}

Beljaars, A. C. M., 1994: The parameterization of surface fluxes in large-scale models under free convection. Quart J. Roy. Meteor. Soc., 121, 255-270.

Bony, S., J. L. Dufresne, H. Le Treut, J. J. Morcrette, and C. Senior, 2004: On dynamic and thermodynamic components of cloud changes. Climate Dyn., 22, 71-86.

Chen, F., and J. Dudhia, 2001: Coupling an advanced land surface-hydrology model with the Penn State-NCAR MM5 modeling system. Part I: Model implementation and sensitivity. Mon. Wea. Rev., 129, 569-585.

Chiriaco, M., H. Chepfer, V. Noel, A. Delaval, M. Haeffelin, P. Dubuisson, and P. Yang, 2004: Improving retrievals of cirrus cloud particle size coupling lidar and three-channel radiometric techniques. Mon. Wea. Rev., 132, 1684-1700.

Collis, R. T., and P. B. Russel, 1976: Laser Monitoring of the Atmosphere. Springer-Verlag.

Doutriaux-Boucher, M., and J. Quaas, 2004: Evaluation of cloud thermodynamic phase parameterizations in the LMDZ GCM by using POLDER satellite data. Geophys. Res. Lett., 31, L06126, doi:10.1029/2003GL019095.

Dudhia, J., 1989: Numerical study of convection observed during the Winter Monsoon Experiment using a mesoscale twodimensional model. J. Atmos. Sci., 46, 3077-3107.

_ 1993: A nonhydrostatic version of the Penn State-NCAR Mesoscale Model: Validation tests and simulation of an Atlantic cyclone and cold front. Mon. Wea. Rev., 121, 14931513.

Fletcher, N. H., 1962: The Physics of Rain Clouds. Cambridge University Press, 386 pp.

Grell, G., 1993: Prognostic evaluation of assumptions used by cumulus parameterizations. Mon. Wea. Rev., 121, 764-787.

Guichard, F., D. B. Parsons, J. Dudhia, and J. Bresch, 2003: Evaluating mesoscale model predictions of clouds and radia- tion with SGP ARM data over a seasonal timescale. Mon. Wea. Rev., 131, 926-944.

Hack, J. J., B. A. Boville, B. P. Briegleb, J. T. Kiehl, P. J. Rasch, and D. L. Williamson, 1993: Description of the NCAR Community Climate Model (CCM2). NCAR Tech. Note NCAR/ TN-382+STR, National Center for Atmospheric Research, Boulder, CO, 108 pp.

Haeffelin, M., and Coauthors, 2004: SIRTA, a ground-based atmospheric observatory for clouds and aerosols research. Ann. Geophys., 23, 253-275.

Heckman, S. T., and W. R. Cotton, 1993: Mesoscale numerical simulation of cirrus clouds-FIRE case study and sensitivity analysis. Mon. Wea. Rev., 121, 2264-2284.

Heymsfield, A. J., and C. M. R. Platt, 1984: A parameterization of the particle size spectrum of ice clouds in term of the ambient temperature and the ice water content. J. Atmos. Sci., 41, 846-855.

— cloud water content in general circulation models. J. Atmos. Sci., 47, 1865-1877.

—, S. Lewis, A. Bansemer, J. Iaquinta, L. M. Miloshevich, M. Kajikawa, C. Twohy, and M. R. Poellot, 2002: A general approach for deriving the properties of cirrus and stratiform ice cloud particles. J. Atmos. Sci., 59, 3-29.

Jakob, C., R. Pincus, C. Hannay, and K. M. Xu, 2004: Use of cloud radar observations for a model evaluation: A probabilistic approach. J. Geophys. Res., 109, D03202, doi:10.109/ 2003JD003473.

Kessler, E., III, 1969: On the Distribution and Continuity of Water Substance in Atmospheric Circulations. Meteor. Monogr., No. 32, Amer. Meteor. Soc., 84 pp.

Khvorostyanov, V. I., and K. Sassen, 1998: Cirrus cloud simulation using explicit microphysics and radiation. Part I: Model description. J. Atmos. Sci., 55, 1808-1821.

Krueger, S. K., G. T. McLean, and Q. Fu, 1995a: Numerical simulation of the stratus-to-cumulus transition in the subtropical marine boundary layer. Part I: Boundary layer structure. $J$. Atmos. Sci., 52, 2839-2850.

,$- \ldots$, and $-1995 \mathrm{~b}$ : Numerical simulation of the stratusto-cumulus transition in the subtropical marine boundary layer. Part II: Boundary layer circulation. J. Atmos. Sci., 52, 2851-2868.

Lafore, J. P., and Coauthors, 1998: The Meso-NH Atmospheric Simulation System. Part I: Adiabatic formulation and control simulations. Ann. Geophys., 16, 90-109.

Levkov, L., B. Rockel, H. Kapitza, and E. Raschke, 1992: 3D mesoscale numerical studies of cirrus and stratus clouds by their time and space evolution. Beitr. Phys. Atmos., 65, 35-57.

,,-- H. Schiller, and L. Kornblueh, 1998: 3-D simulation of clouds with subgrid fluctuations of temperature and humidity. Atmos. Res., 47-48, 327-341.

Lin, R. F., 1997: A numerical study of the evolution of nocturnal cirrus by a two-dimensional model with explicit microphysics. Ph.D. thesis, The Pennsylvania State University, 199 pp.

Liou, K. N., 1986: Influence of cirrus clouds on weather and climate processes: A global perspective. Mon. Wea. Rev., 114, 1167-1199.

_ 2002: An Introduction to Atmospheric Radiation. International Geophysics Series, Vol. 84, Academic Press, 175 pp.

Liu, Y., F. Chen, T. Warner, and S. Swerdlin, 2004: Improvements to surface flux computations in the MRF PBL scheme, and refinements to urban processes in the NOAH land-surface model with the NCAR/ATEC real-time FDDA and forecast 
system. Proc. Fifth WRF/MM5 Users Workshop, Boulder, CO.

Marshall, J., and W. Palmer, 1948: The distribution of raindrops with size. J. Meteor., 5, 165-166.

Minnis, P., D. P. Garber, and D. F. Young, 1998: Parameterization of reflectance and effective emittance for satellite remote sensing of cloud properties. J. Atmos. Sci., 55, 3313-3339.

Noel, V., H. Chepfer, G. Ledanois, and P. H. Flamant, 2001: Computation of single-scattering matrix for non-spherical particles randomly or horizontally oriented in space. Appl. Opt., 40, 4365-4375.

, A. Delaval, and P. H. Flamant, 2002: Classification of particle effective shape ratios in cirrus clouds based on lidar depolarization ratio. Appl. Opt., 41, 4245-4257.

Platt, C. M. R., 1973: Lidar and radiometric observations of cirrus clouds. J. Atmos. Sci., 30, 1191-1204.

Protat, A., J. Delanoë, D. Bouniol, and M. Haeffelin, 2004: Dynamical, microphysical and radiative properties of ice clouds using Doppler cloud radar measurements. Proc. Third European Radar Conf., Visbi, Sweden.

Reisner, J., R. M. Rasmussen, and R. T. Bruintjes, 1998: Explicit forecasting of supercooled liquid water in winter storms using the MM5 mesoscale model. Quart. J. Roy. Meteor. Soc., 124, 1071-1107.

Sassen, K., 1991: The polarization lidar technique for cloud research: A review and current assessment. Bull. Amer. Meteor. Soc., 72, 1848-1866.

Tinel, C., J. Testud, J. Pelon, R. Hogan, A. Protat, J. Delanoë, and D. Bouniol, 2005: The retrieval of ice-cloud properties from cloud radar and lidar synergy. J. Appl. Meteor., 44, 860-875.

Troën, I., and L. Mahrt, 1986: A simple model of the atmospheric boundary: Sensitivity to surface evaporation. Bound.-Layer Meteor., 37, 129-148.

Ulaby, F. T., R. K. Moore, and A. K. Fung, 1943: Microwave Remote-Sensing Active and Passive. Vol. 1, Microwave RemoteSensing Fundamentals and Radiometry. Artech House.

Walko, R. L., W. R. Cotton, M. P. Meyers, and J. Y. Harrington, 1995: New RAMS cloud microphysics parameterization. Part I: The single-moment scheme. Atmos. Res., 38, 29-62.

Xu, K. M., A. Arakawa, and S. K. Krueger, 1992: The macroscopic behavior of cumulus ensembles simulated by a cumulus ensemble model. J. Atmos. Sci., 49, 2402-2420.

Zurovac-Jevtic, D., and G. J. Zhang, 2003: Development and test of a cirrus parameterization scheme using NCAR CCM3. $J$. Atmos. Sci., 60, 1325-1344. 